ARTICLE

\title{
Orbitofrontal control of visual cortex gain promotes visual associative learning
}

\author{
Dechen Liu (1) 1,2, Juan Deng ${ }^{1}$, Zhewei Zhang (1) 1,2,3, Zhi-Yu Zhang ${ }^{1,2}$, Yan-Gang Sun (i) 1,4, Tianming Yang (1) ${ }^{3,4} \&$ \\ Haishan Yao (1) 1,4凶
}

The orbitofrontal cortex (OFC) encodes expected outcomes and plays a critical role in flexible, outcome-guided behavior. The OFC projects to primary visual cortex (V1), yet the function of this top-down projection is unclear. We find that optogenetic activation of OFC projection to $\mathrm{V} 1$ reduces the amplitude of $\mathrm{V} 1$ visual responses via the recruitment of local somatostatin-expressing (SST) interneurons. Using mice performing a Go/No-Go visual task, we show that the OFC projection to V1 mediates the outcome-expectancy modulation of V1 responses to the reward-irrelevant No-Go stimulus. Furthermore, V1-projecting OFC neurons reduce firing during expectation of reward. In addition, chronic optogenetic inactivation of OFC projection to V1 impairs, whereas chronic activation of SST interneurons in V1 improves the learning of Go/No-Go visual task, without affecting the immediate performance. Thus, OFC top-down projection to $\mathrm{V} 1$ is crucial to drive visual associative learning by modulating the response gain of $\mathrm{V} 1$ neurons to non-relevant stimulus.

\footnotetext{
${ }^{1}$ Institute of Neuroscience, State Key Laboratory of Neuroscience, CAS Center for Excellence in Brain Science and Intelligence Technology, Chinese Academy of Sciences, Shanghai 200031, China. ${ }^{2}$ University of Chinese Academy of Sciences, Beijing 100049, China. ${ }^{3}$ Institute of Neuroscience, Key Laboratory of Primate Neurobiology, CAS Center for Excellence in Brain Science and Intelligence Technology, Chinese Academy of Sciences, Shanghai 200031, China.

${ }^{4}$ Shanghai Center for Brain Science and Brain-Inspired Intelligence Technology, Shanghai 201210, China. ${ }^{凶}$ email: haishanyao@ion.ac.cn
} 
T he OFC is involved in encoding specific stimulus-reward associations that guide adaptive behavior ${ }^{1-3}$. Studies in rodents and monkeys have demonstrated that the identity and expected values of specific outcomes are represented by activities in the $\mathrm{OFC}^{4-12}$. Lesions or inactivation of the OFC impair behavior guided by outcome expectancy and learning driven by the discrepancy between expected and actual outcomes $^{13-26}$, and degrade the acquisition of Pavlovian trace conditioning task ${ }^{27}$.

Direct output from the OFC to other brain regions, including the basolateral amygdala (BLA), ventral tegmental area (VTA), and striatum, is important for learning and reward-related behavior $^{2,3}$. In OFC-lesioned rats, BLA neurons are impaired in the encoding of cue-outcome association and the developing of outcome-expectant activity ${ }^{28}$. The outcome-expectancy signals in the OFC are necessary for VTA dopamine neurons to calculate reward prediction errors ${ }^{29}$, which are important teaching signals for reinforcement learning ${ }^{30}$. Inactivation of the OFC or disconnection of the OFC from VTA prevents extinction learning in the Pavlovian over-expectation task ${ }^{23,31}$. The VTA-projecting OFC neurons encode long-term memory of cue-reward association, and optogenetic inhibition of these neurons impairs extinction learning and memory ${ }^{27}$. The OFC also connects with sensory cortices ${ }^{32-34}$, including $\mathrm{V}^{32}$. It is unknown how the responses of sensory cortex-projecting OFC neurons are modulated by outcome expectancy, and whether the top-down signals from the OFC to sensory cortices influence learning behavior.

Frontal top-down projections to sensory cortices are known to modulate sensory processing ${ }^{35-37}$, promote accurate perception $^{38}$, and convey predictive signals $s^{39,40}$. Associative learning enhances signals related to stimulus expectation or reward expectation in V1, which may be mediated by top-down projections $^{41}$. Learning also enhances the effect of top-down inputs in modulating V1 responses ${ }^{42}$. However, the causal role of top-down projections to sensory cortices in stimulus-reward associative learning remains unclear.

In this study, we find that activation of OFC top-down projection results in suppression of V1 visual response by activating SST interneurons. In mice performing a Go/No-Go visual task, V1 responses to the reward-irrelevant No-Go stimulus are lower when the mice' outcome expectation is correct than when it is incorrect, and such response modulation is reduced by optogenetic inactivation of OFC projection to V1. Optogenetic tagging of V1-projecting OFC neurons reveals that their responses to the No-Go stimulus are reduced in trials in which mice performed incorrectly. We further show that chronic optogenetic inactivation of OFC projection to V1 slows the learning of Go/No-Go visual behavior. Thus, the OFC projection to V1 plays a key role in filtering out non-relevant visual information to facilitate associative learning.

\section{Results}

Effect of activating OFC top-down projection on V1 responses. We injected Cholera toxin subunit B (CTB) in V1 and found that the retrograde labeled neurons were in the ventrolateral OFC (vlOFC) (Fig. 1a), consistent with the finding in a previous study $^{32}$. By injecting rAAV2-retro-hSyn-Cre in V1 and AAVDIO-EYFP in the OFC, we found that OFC axons terminated in both superficial and deep layers of V1 (Fig. 1b). To examine how OFC top-down projection influences V1 neuronal responses, we expressed excitatory opsin Channelrhodopsin-2 (ChR2) or ChrimsonR in the OFC, and measured V1 responses with and without laser stimulation of OFC axons in mice passively viewing drifting gratings (Fig. 1c). Activating OFC axons in V1 significantly reduced the firing rates of V1 neurons in both anesthetized and awake mice (anesthetized mice: $P=5.2 \times 10^{-6}$, $n=102$ neurons; awake mice: $P=6.53 \times 10^{-5}, n=62$ neurons; Wilcoxon signed rank test, Fig. 1d). When we computed a rate change index as $\left(R_{\text {laser_on }}-R_{\text {laser_off }}\right) /\left(R_{\text {laser_on }}+R_{\text {laser_off }}\right)$, where $R_{\text {laser_on }}$ and $R_{\text {laser_off }}$ represented responses averaged overall orientations for laser-on and laser-off trials, respectively, we found that the index was negative for the majority of V1 neurons in both anesthetized and awake mice (Fig. 1e). For control mice injected with AAV-mCherry (or AAV-EGFP) in the OFC, the laser-induced response reduction was significantly smaller than that for mice injected with AAV-ChR2 (or AAV-ChrimsonR) (Supplementary Fig. 1a). After blocking antidromic spikings of OFC neurons with tetrodotoxin in the OFC, the response reduction in V1 neurons induced by activating OFC axons was still significant $\left(P=2.33 \times 10^{-12}, n=118\right.$ neurons from awake mice, Wilcoxon signed rank test, Fig. $1 \mathrm{f}-\mathrm{h}$ ), indicating that the laser-induced response reduction was mediated directly by OFC projection to V1, rather than through antidromic activation of indirect pathways. Although the response amplitude was clearly reduced, the orientation selectivity of V1 neurons was not affected by activation of OFC axons in V1 (anesthetized mice: $P=0.36$, $n=102$ neurons; awake mice: $P=0.99, n=62$ neurons; Wilcoxon signed rank test, Fig. 1i).

To dissect the circuit mechanism underlying V1 response modulation by the OFC top-down projection, we infected the OFC neurons with AAV-ChR2 and performed whole-cell recordings from $\mathrm{V} 1$ neurons in acute slices of $\mathrm{V} 1$ containing ChR2-expressing OFC axons. Photostimulation of OFC axons evoked both excitatory and inhibitory postsynaptic currents (EPSCs and IPSCs) in the recorded neurons (Fig. 2a). The onset latencies of the EPSCs were shorter than those of the IPSCs (Fig. 2b), which were blocked by $\gamma$-aminobutyric acid type A $\left(\mathrm{GABA}_{\mathrm{A}}\right)$ receptor antagonist picrotoxin (Fig. 2c). We next tested whether IPSCs evoked by activation of OFC axons were feedforward inhibition. We bath applied an a-amino-3hydroxy-5-methyl-4-isoxazole propionic acid (AMPA) receptor antagonist NBQX, and found that the amplitudes of both EPSCs and IPSCs were reduced (Fig. 2d), indicating that the IPSCs were due to feedforward inhibition generated by local inhibitory neurons in V1. By injecting CTB in V1 of GAD67-GFP mice, we found that the retrograde labeling of OFC neurons did not overlap with the GFP-positive neurons in the OFC (Fig. 2e), confirming that the V1-projecting OFC neurons were not GABAergic.

We next examined which subtype of inhibitory neurons mediates the effect of activating OFC axons on V1 responses. By using rabies virus (RV)-mediated monosynaptic retrograde tracing ${ }^{43}$, we found that $\mathrm{V} 1$ interneurons expressing parvalbumin (PV), SST and vasoactive intestinal peptide (VIP) all received direct innervation from neurons in the vlOFC (Supplementary Fig. 2). Consistently, slice recording showed that optogenetic stimulation of OFC axons evoked EPSCs in three different subtypes of interneurons as well as pyramidal neurons in V1 (Fig. 2f). As the latencies of EPSCs were short (Fig. 2f), the EPSCs were likely evoked by direct excitatory drive from the OFC.

We further used fiber photometry to measure the activities of three subtypes of V1 interneuron in vivo in response to optogenetic activation of OFC neurons (Fig. 2g). For these mice, we expressed ChR2 in the OFC and calcium indicator GCaMP6s in PV, SST, or VIP interneurons in V1, respectively (Fig. 2h). During the experiment, the mice were awake but were not viewing visual stimulus. We found that optogenetic activation of OFC neurons caused increase in calcium signals in SST interneurons, but reduction in calcium signals in PV and VIP interneurons in V1 (Fig. 2i, j). Since the latency of laser-evoked peak responses for PV interneurons was longer than that for SST 
a
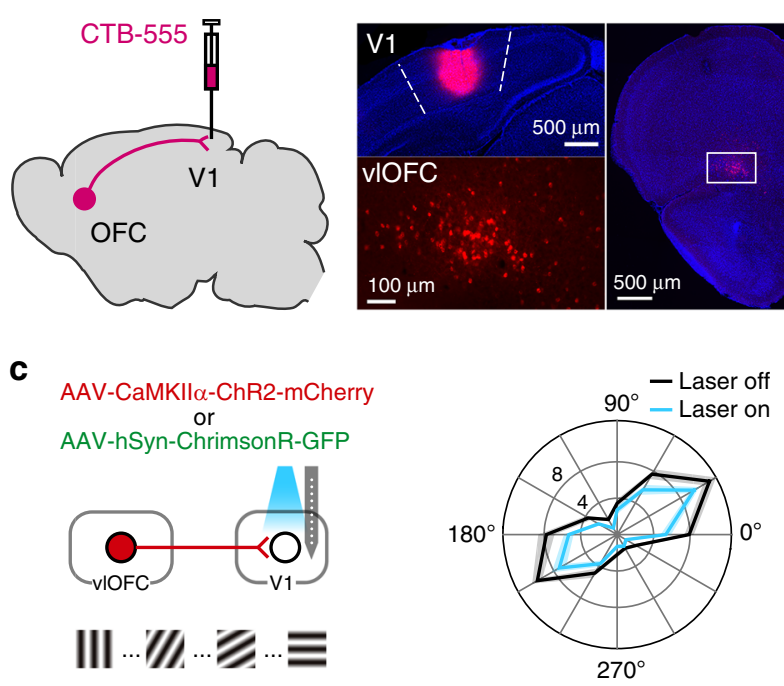

f

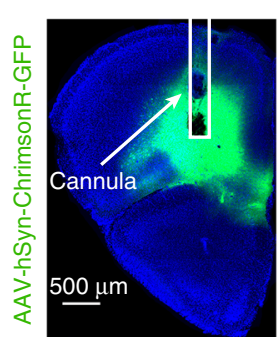

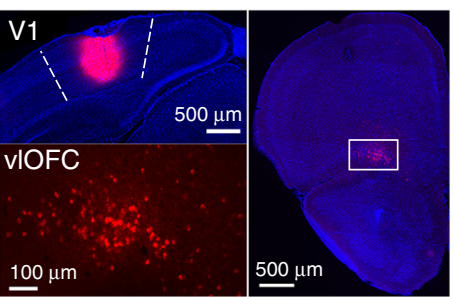

g

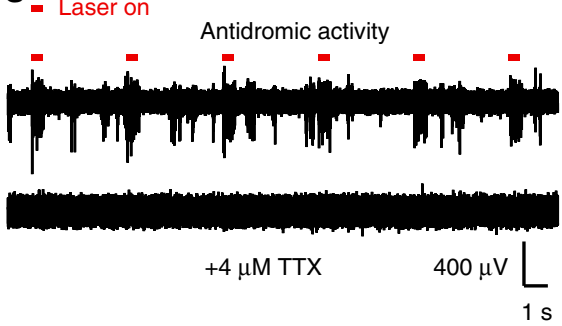

b

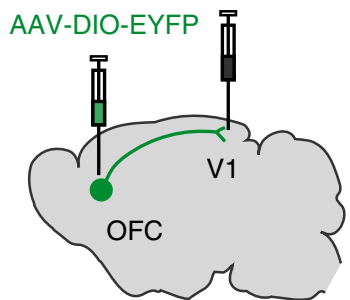

d

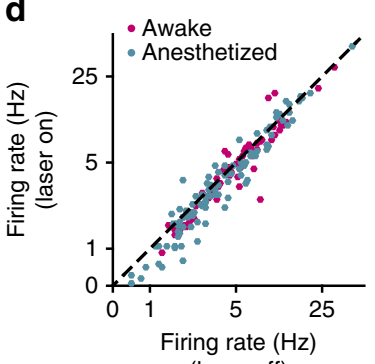

(laser off)

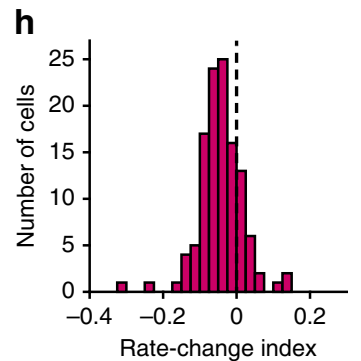

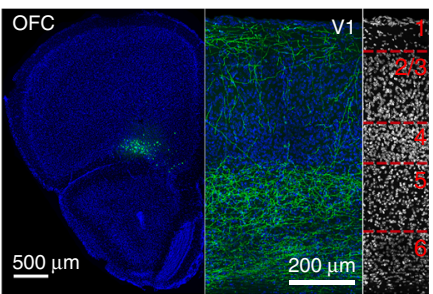

e

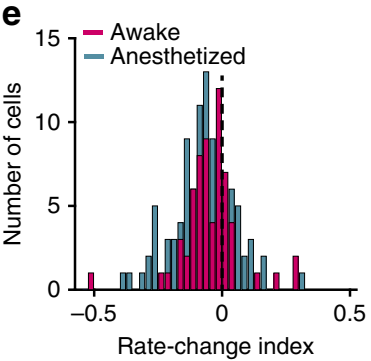

i

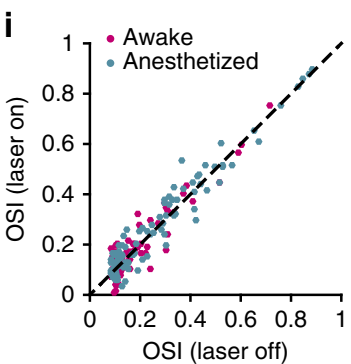

Fig. 1 Activating OFC projection to V1 reduces response amplitude of V1 neurons. a Left, schematic of CTB injection in V1. Right, Representative fluorescence images of CTB injection in V1 and retrograde labeled neurons in the ventrolateral OFC. $\mathbf{b}$ Left, virus strategy to visualize OFC axons in V1. Right, representative fluorescence images of V1-projecting OFC neurons and their terminals in V1. The experiments in $\mathbf{a}$ and $\mathbf{b}$ were repeated $>3$ times with similar results. $\mathbf{c}$ Left, schematic of measuring V1 visual responses with and without activating OFC axons. Right, tuning curves of a V1 neuron with (blue) and without (black) laser stimulation of OFC axons. d Mean firing rate (firing rate averaged overall orientations) of V1 neurons with laser-on vs. laser-off. Anesthetized mice (blue): $P=5.2 \times 10^{-6}, n=102$ neurons; awake mice (magenta): $P=6.53 \times 10^{-5}, n=62$ neurons. e Distribution of rate change indexes for V1 neurons in anesthetized $\left(P=2.6 \times 10^{-8}\right)$ and awake mice $\left(P=3.81 \times 10^{-5}\right)$. $\mathbf{f}$ TTX was infused to the OFC, in which AAV-hSyn-ChrimsonR-GFP had been injected, to block the antidromic spikes induced by laser stimulation in V1. White rectangle shows the placement of the cannula. $\mathbf{g}$ TTX infusion into the OFC abolished multi-unit activity in the OFC evoked by laser stimulation in V1. Upper trace, without TTX; Lower trace, with TTX infusion into the OFC. h Distribution of rate change indexes of $\mathrm{V} 1$ neurons recorded with TTX infusion into the OFC. $P=2.33 \times 10^{-12}, n=118$ neurons from awake mice. $\mathbf{i}$, Orientation selectivity index (OSI) with laser-on vs. laser-off. Anesthetized mice (blue): $P=0.36, n=102$; awake mice (magenta): $P=0.99, n=62$. For d, e, h, i AAV-CaMKIl $\alpha$-hChR2 (H134R)-mCherry and AAV-hSyn-ChrimsonR-GFP were injected in the OFC for anesthetized and awake mice, respectively. Wilcoxon two-sided signed rank test. For $\mathbf{d}, \mathbf{e}, \mathbf{h}, \mathbf{i}$ source data are provided as a Source Data file. Shadings, mean \pm s.e.m.

interneurons (Fig. 2k), the reduced PV neuronal responses were likely to be attributed to inhibition caused by SST interneuron activation $^{44}$. Early activated population of SST interneurons could also inhibit VIP interneurons ${ }^{44}$, causing their activity reduction. Therefore, OFC stimulation in vivo preferentially activated SST interneurons in $\mathrm{V} 1$, providing a circuit mechanism for top-down modulation of $\mathrm{V} 1$ responses by the OFC.

OFC projection modulates $\mathrm{V} 1$ responses to irrelevant stimulus. To examine whether inactivating the OFC projection to $\mathrm{V} 1$ influences V1 neuronal responses, we expressed inhibitory opsin Jaws in the OFC. By recording V1 neurons from awake mice passively viewing drifting gratings, we found that inactivating the OFC projection to V1 did not cause significant change in V1 responses as compared to the control mice $(P=0.17$, Wilcoxon rank sum test, Supplementary Fig. 1b, c).

We next wondered whether the OFC projection to V1 may function during task engagement to suppress V1 responses to non-relevant visual stimulus. To test this hypothesis, we trained head-fixed mice to perform a Go/No-Go visual task (Fig. 3a, b), in which a vertical grating (the "Go" stimulus) and a horizontal grating (the "No-Go" stimulus) were associated with water reward and no reward, respectively. In each trial, the duration of stimulus presentation included a waiting period, during which licking had no consequence, and an answer period (Fig. 3a). For a Go trial, licking within the answer period was rewarded with water (hit). For a No-Go trial, licking (false alarm, FA) within the answer period was neither rewarded nor punished, and withholding licking within the answer period represented correct rejection (CR). During the intertrial interval (ITI), the screen was blank and licking was punished with a longer ITI. The training of the Go/No-Go task was preceded by 2 sessions of conditioning, in which the mouse learned to lick within the answer period after the presentation of a Go stimulus. We found that the latency of the first lick after stimulus onset increased with training, and the ITI decreased over sessions (Fig. 3b, Supplementary Fig. 3), indicating that the mice gradually understood the task structure. 
a

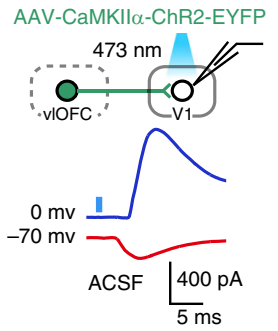

b

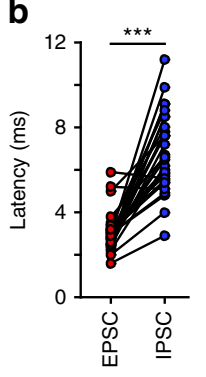

c

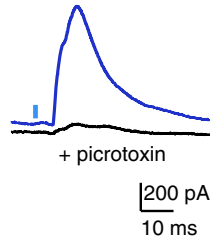

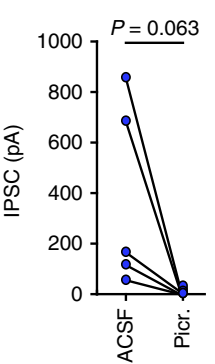

d
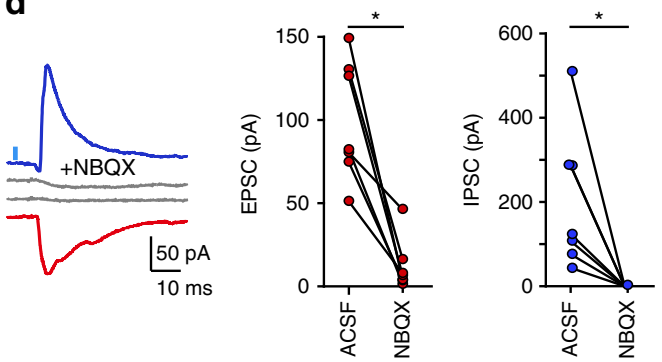

e

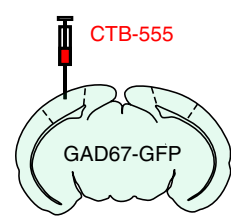

vIOFC

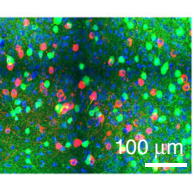

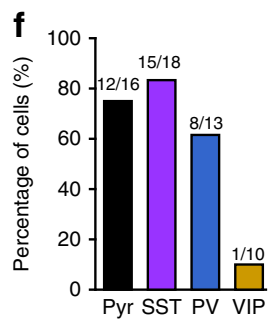

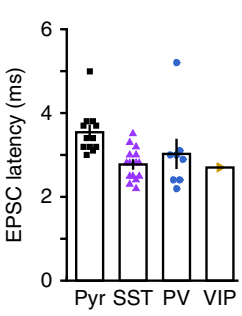

i
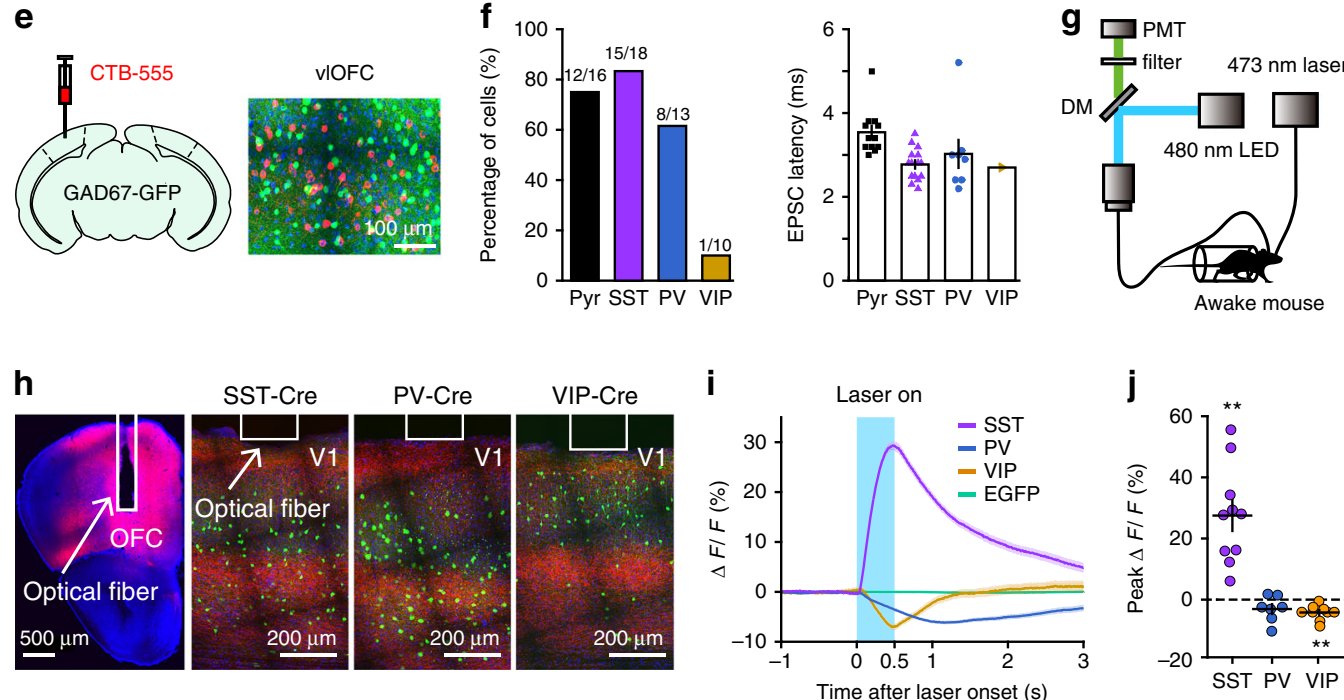

AAV-CaMKIl $\alpha-C h R 2-m$ Cherry AAV-hSyn-FLEX-GCaMP6s
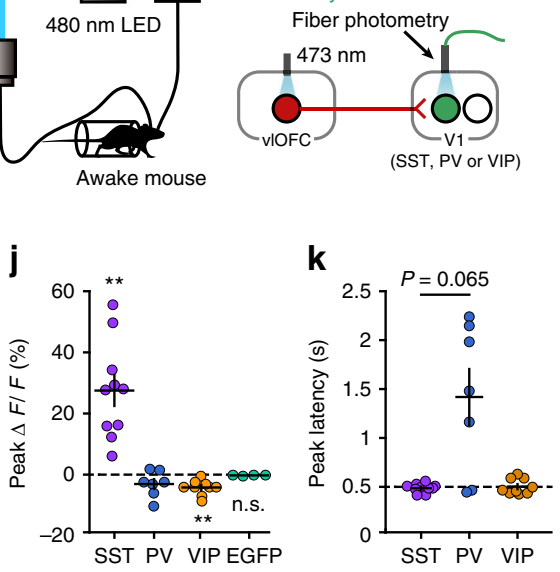

Fig. 2 Activating OFC axons activates V1 inhibitory interneurons. a Photostimulation of OFC projection to V1 evoked both EPSCs (red) and IPSCs (blue) in a V1 neuron. $\mathbf{b}$ The EPSC latencies vs IPSC latencies ( ${ }^{\star \star \star} P=2.34 \times 10^{-6}, n=30$, in which $n=7,12,1,1$, and 9 for pyramidal, SST, PV, VIP, and unidentified neurons, respectively). c Left, GABA $A$ receptor antagonist (picrotoxin, $30 \mu \mathrm{M}$ ) application blocked IPSCs in a V1 neuron. Black, IPSCs after picrotoxin application. Right, IPSCs amplitudes before and after picrotoxin application $(P=0.063, n=5)$. d Left, AMPA receptor antagonist (NBQX, $10 \mu \mathrm{M})$ application blocked both EPSCs and IPSCs in a V1 neuron. Gray, EPSCs and IPSCs after NBQX application. Middle and right, EPSCs (IPSCs) amplitudes before and after NBQX application ( $P=0.016$ and $n=7$ neurons for both EPSCs and IPSCs). e CTB was injected in V1 of a GAD67-GFP mouse. Retrograde labeling of OFC neurons did not overlap with GFP-positive OFC neurons. $\mathbf{f}$ Left, fraction of pyramidal (Pyr) and inhibitory interneurons showing laserevoked EPSCs. Right, EPSC latency for each type of neuron. $\mathbf{g}$ Schematic of the strategy to record activities of specific interneuron type in V1 evoked by OFC activation. $\mathbf{h}$ Fluorescence image of OFC in which AAV-CaMKIl $\alpha$-ChR2-mCherry was injected, and those of V1 injected with AAV-hSyn-FLEX-GCaMP6s from a SST-Cre, PV-Cre, and VIP-Cre mouse, respectively. White rectangles, placement of the optic fiber. The results were replicated $>6$ times.

i Representative $\Delta F / F$ signals from a SST-Cre, PV-Cre, VIP-Cre mouse, and a C57BL/6 mouse (AAV-hSyn-EGFP injected in V1), respectively. Blue, OFC stimulation. j Amplitude of peak $\Delta F / F$. SST: ${ }^{\star} P=0.002, n=10$ mice; PV: $P=0.078, n=7$ mice; VIP: ${ }^{\star \star} P=0.004, n=9$ mice; EGFP: $P=0.13, n=4$ mice. $\mathbf{k}$ Latency of peak $\Delta F / F$. SST vs PV: $P=0.065$ (Wilcoxon two-sided rank sum test). Wilcoxon two-sided signed rank test for $\mathbf{b}, \mathbf{c}, \mathbf{d}, \mathbf{j}$. For $\mathbf{b}-\mathbf{d}, \mathbf{f}$, and $\mathbf{i}-\mathbf{k}$, source data are provided as a Source Data file. Shadings and error bars, mean \pm s.e.m.

Over 11 training sessions of the Go/No-Go task, the hit rate remained high throughout all sessions, whereas the CR rate and discriminability $\left(d^{\prime}\right)$ increased with training (Fig. 3c, d). Thus, the learning of the task depended on the improvement of CR for the reward-irrelevant No-Go stimulus.

We performed single-unit extracellular recordings from V1 in behaving mice that had been trained for at least 5 days. We first compared the responses of $\mathrm{V} 1$ neurons to the Go and No-Go stimuli (Fig. 3e, f). We defined a selectivity index (SI) as $\left(\mathrm{R}_{\mathrm{Go}}-\right.$ $\left.\mathrm{R}_{\mathrm{No}-\mathrm{Go}}\right) /\left(\mathrm{R}_{\mathrm{Go}}+\mathrm{R}_{\mathrm{No}-\mathrm{Go}}\right)$, where $\mathrm{R}_{\mathrm{Go}}$ and $\mathrm{R}_{\mathrm{No}-\mathrm{Go}}$ are firing rates to the Go and No-Go stimuli during the waiting period, respectively. The SIs of V1 neurons were significantly larger than zero $\left(P=2.2 \times 10^{-12}\right.$, Wilcoxon signed rank test) and significantly larger than those in untrained mice passively viewing the stimulus $\left(P=4.6 \times 10^{-4}, n=394\right.$ neurons from trained mice and 160 neurons from untrained mice, Wilcoxon rank sum test, Fig. 3g, h), indicating a higher response to the Go stimulus than to the No-Go stimulus, consistent with previous reports ${ }^{41}$. While the SIs for the early response component $(<100 \mathrm{~ms})$ were not significantly different from zero, the SIs for the late response component (>100 ms) were significantly larger than zero (Fig. 3i). We also compared the responses of V1 neurons to the No-Go stimulus between $\mathrm{CR}$ and FA trials (Fig. 3j, k). We defined a modulation index $(\mathrm{MI})$ as $\left(\mathrm{R}_{\mathrm{CR}}-\mathrm{R}_{\mathrm{FA}}\right) /\left(\mathrm{R}_{\mathrm{CR}}+\mathrm{R}_{\mathrm{FA}}\right)$, where $\mathrm{R}_{\mathrm{CR}}$ and $R_{F A}$ are firing rates to the No-Go stimulus during the waiting period in CR and FA trials, respectively. We found that the MIs were significantly smaller than zero for the population of V1 neurons $\left(P=1.46 \times 10^{-42}, n=394\right.$ neurons, Wilcoxon signed rank test, Fig. 31). For neurons preferring the Go stimulus (SI $>0$, $n=270$ ) and those preferring the No-Go stimulus (SI $<0, n=$ 124), the MIs were both significantly smaller than zero (Fig. $3 \mathrm{~m}$ ). Similar to the SI, the magnitude of MI was significant for the late but not for the early response component (Fig. 3n). In addition, we classified the neurons into broad-spiking and narrow-spiking cells ${ }^{45,46}$, which correspond to putative excitatory and putative inhibitory neurons, respectively. We found that the SIs of both cell types were significantly larger than zero, and the MIs of both cell types were significantly smaller than zero (Supplementary 
a
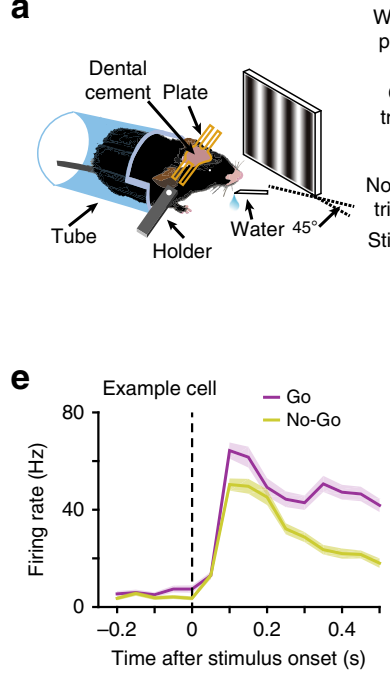

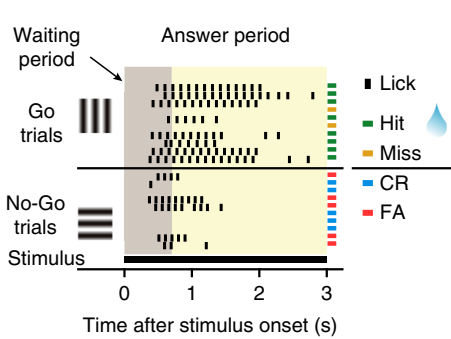

f

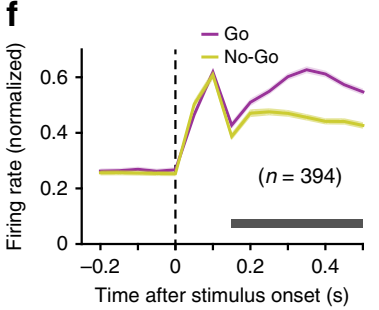

b

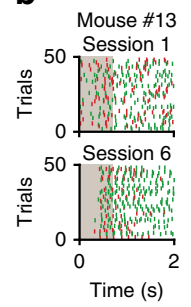

C

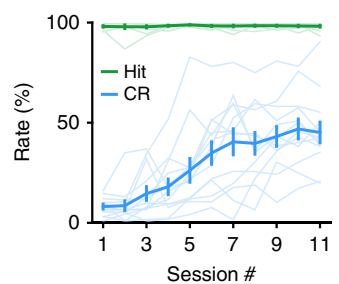

d

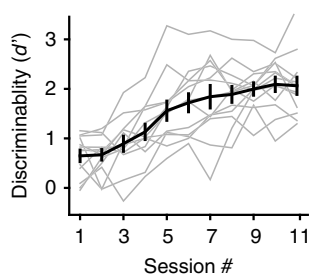

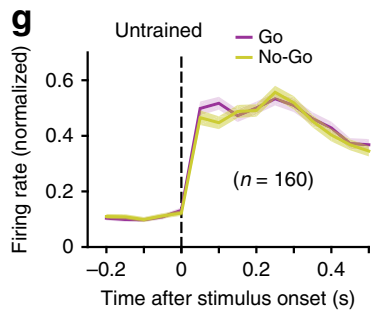

h

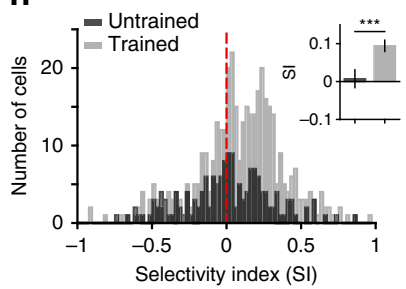

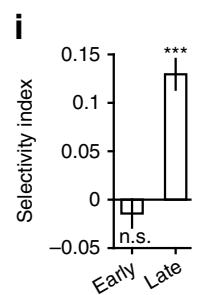

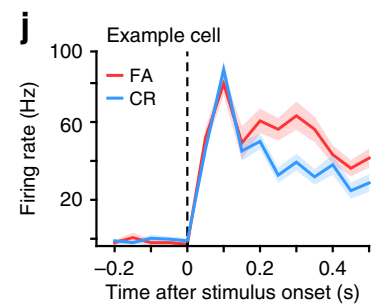

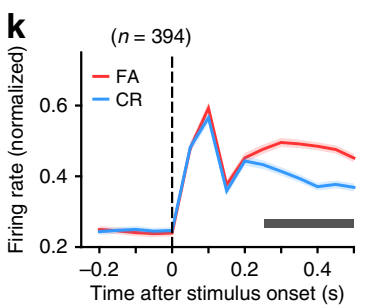
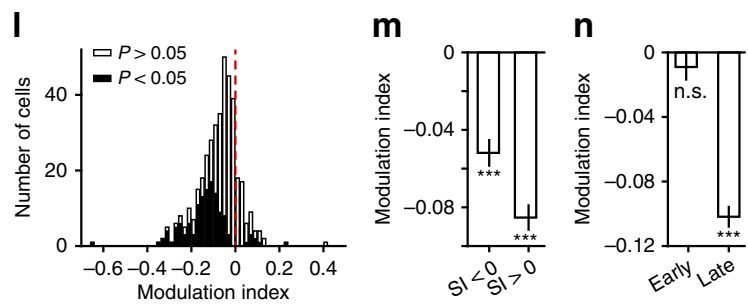

Fig. 3 Responses of $\mathbf{V} \mathbf{1}$ neurons in Go/No-Go visual task. a Behavioral setup and task structure. $\mathbf{b}$ Example lick rasters in two sessions $\mathbf{c}$ Hit rate (green), $F_{(2.72,32.62)}=0.43, P=0.71 ; C R$ rate (blue), $F_{(3.36,40.28)}=23.57, P=2.1 \times 10^{-9}$. d Discriminability, $F_{(4.49,53.86)}=33.34, P=1.1 \times 10^{-14}$. Each thin line represents a mouse. One-way repeated measures ANOVA with the Greenhouse-Geisser correction for $\mathbf{c}$ and $\mathbf{d}$. e Responses of an example neuron to Go and No-Go stimuli. f Normalized responses of V1 neurons in Go and No-Go trials. Horizontal bar, timepoints with $P<0.05$, two-way repeated measures ANOVA $F_{(1,393)}=55.7, P=5.5 \times 10^{-13}$ followed by Sidak's multiple comparisons test. $\mathbf{g}$ Responses of $\mathrm{V} 1$ neurons from untrained mice $(P=0.56$, two-way repeated measures ANOVA $\left.F_{(1,159)}=0.33\right)$. h Distribution of SIs. Light gray, trained mice, $P=2.2 \times 10^{-12}, n=394$ neurons. Dark gray, untrained mice, $P=$ $0.62, n=160$ neurons. Wilcoxon two-sided signed rank test. Inset, Sls from untrained and trained mice. ${ }^{\star \star \star} P=4.6 \times 10^{-4}$, Wilcoxon two-sided rank sum test. $\mathbf{i}$ Sls for early $(P=0.51)$ and late $\left({ }^{\star \star \star} P=2.1 \times 10^{-16}\right)$ response components. $n=394$, Wilcoxon two-sided signed rank test. $\mathbf{j}$ Responses of an example neuron to No-Go stimulus in FA and CR trials. $\mathbf{k}$ Normalized responses of V1 neuron to No-Go stimulus in FA and CR trials. Horizontal bar, timepoints with $P<0.05$, two-way repeated measures ANOVA $F_{(1,393)}=222.22, P<1 \times 10^{-15}$ followed by Sidak's multiple comparisons test. I Distribution of Mls $(P=$ $1.46 \times 10^{-42}, n=394$ neurons, Wilcoxon two-sided signed rank test). Black and open bars, significant and not significant Mls. m Mls for neurons with SI > $0(n=270)$ and with $\mathrm{SI}<0(n=124)\left({ }^{\star \star \star} P<2 \times 10^{-10}\right.$, Wilcoxon two-sided signed rank test). $\mathbf{n}$ Mls for early $(P=0.06)$ and late $\left({ }^{\star \star \star} P=5.5 \times 10^{-47}\right)$ response components. $n=394$, Wilcoxon two-sided signed rank test. For c-n, source data are provided as a Source Data file. Shadings and error bars, mean \pm s.e.m.

Fig. 4). These results suggest that, during the waiting period, the responses of V1 neurons to the reward-irrelevant No-Go stimulus were lower than those to the Go stimulus, and the responses to the No-Go stimulus were lower in trials in which the animals performed correctly than in trials performed incorrectly.

The stimulus selectivity and response modulation of V1 neurons during the waiting period could be attributed to multiple factors, including movement, reward expectation, and top-down modulation ${ }^{41,47}$. We first analyzed whether V1 neurons exhibit licking-movement-related activity. We computed Pearson's correlation coefficients between spike rate of V1 neurons and lick rate during the waiting period. The distribution of correlation was not significantly different from zero (Supplementary Fig. 5a). For the lick-triggered spike histogram computed using licks in the waiting period, we did not observe increase in V1 responses after the time of lick (Supplementary Fig. 5b). We also analyzed the responses during the waiting period using those trials in which no lick occurred within the first $0.5 \mathrm{~s}$ following stimulus onset. For these no-lick trials, the responses to the Go stimulus were significantly larger than those to the No-Go stimulus, and the responses to the No-Go stimulus remained significantly lower in CR than in FA trials (Supplementary Fig. $5 c-f$ ). Thus, the response modulation in V1 could not be attributed to licking movement.

We next examined whether the OFC top-down projection plays a role in the modulation of V1 responses in the Go/No-Go task. We expressed inhibitory opsin Jaws in the OFC, and recorded from V1 neurons with and without optogenetic inactivation of OFC axons in behaving mice. Laser stimulation was turned on $100-\mathrm{ms}$ before or at stimulus onset, covering the duration of stimulus presentation, and laser-on and laser-off blocks were interleaved. For one group of mice, laser stimulation was applied during Go trials (Fig. $4 a-d$ ). We found that inactivating OFC axons in V1 during Go trials neither affect V1 responses to the Go stimulus during the waiting period (Fig. 4c), nor the behavioral performance of the mice (Fig. 4d). For another group of mice, laser stimulation was applied during No-Go trials (Fig. $4 \mathrm{e}-\mathrm{p}$ ). Inactivating OFC axons during No-Go trials significantly increased the responses of V1 neurons to the No-Go stimulus during the waiting period $(P=0.01, n=169$ 
a

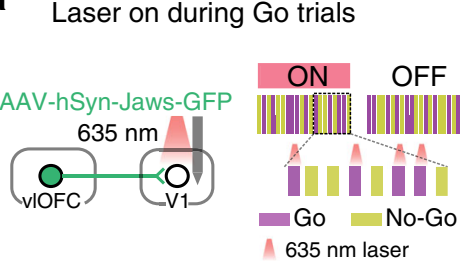

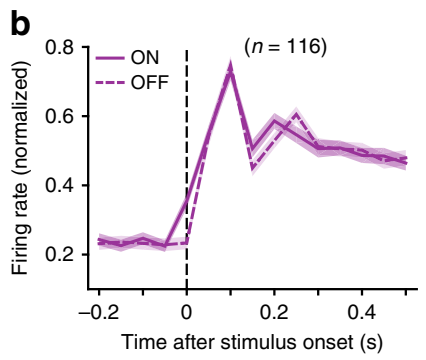

c

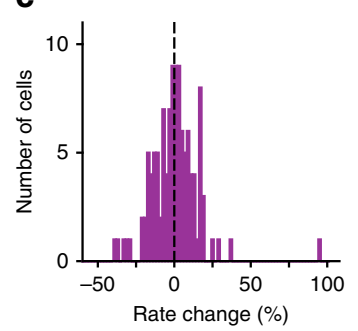

d

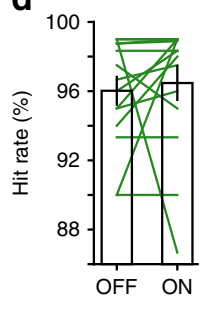

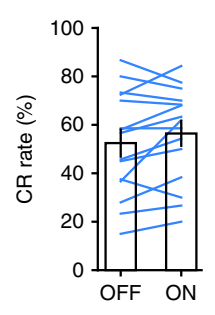
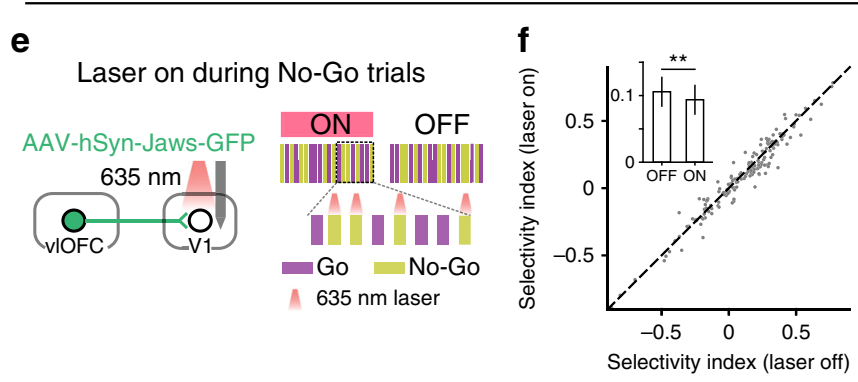
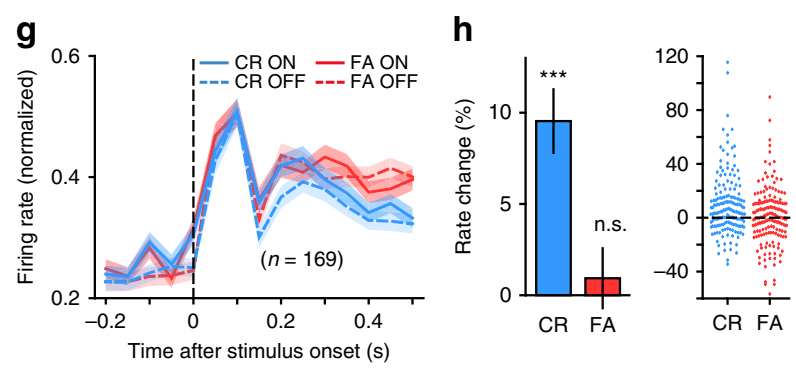
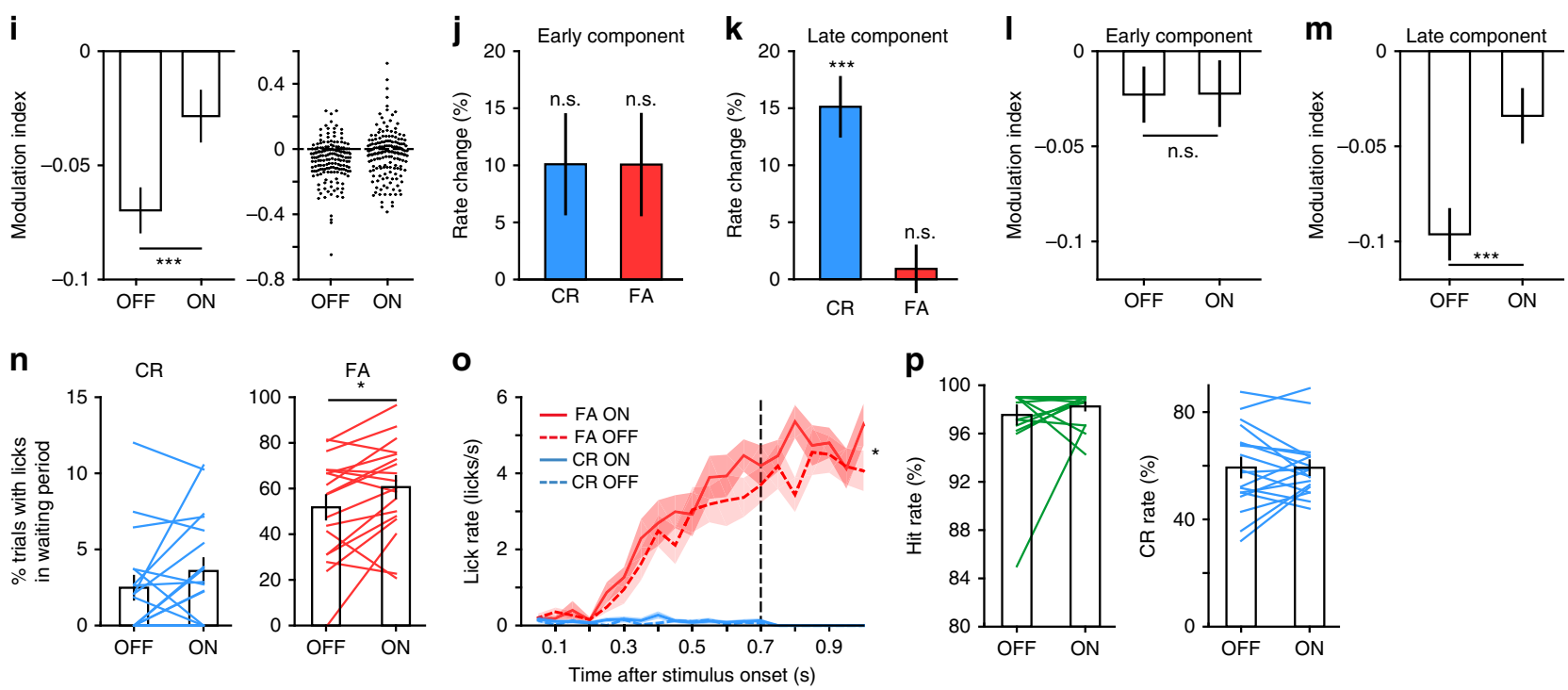

Fig. 4 Effect of inactivating OFC top-down projection on V1 responses. a Schematic of laser stimulation. $\mathbf{b}$ Normalized responses to Go stimulus with and without inactivating OFC axons. c Distribution of rate change induced by inactivating OFC axons during Go trials. $P=0.87, n=116$ neurons. d Inactivating OFC axons during Go trials did not affect hit rate $(P=0.26)$ or CR rate $(P=0.11, n=15$ sessions from seven mice). e Schematic of laser stimulation. $\mathbf{f}$ Inactivating OFC axons during No-Go trials significantly reduced the SI of V1 neurons ( ${ }^{\star} P=0.007, n=169$ ). $\mathbf{g}$ Normalized responses to No-Go stimulus in FA and CR trials, respectively, with and without laser stimulation. $\mathbf{h}$ Inactivating OFC axons during No-Go trials increased the responses of $\mathrm{V} 1$ neurons in $\mathrm{CR}\left({ }^{\star \star \star} P=1.02 \times 10^{-6}\right)$ but not in FA trials $(P=0.66) . n=169$ neurons. $\mathbf{i}$ Inactivating OFC axons during No-Go trials significantly reduced $\mathrm{Ml}$ amplitude ( ${ }^{\star \star \star} P=6.4 \times 10^{-4}, n=169$ neurons). j Rate change for early response component $(C R, P=0.18 ; F A, P=0.77)$. $\mathbf{k}$ Rate change for late response component $\left(C R,{ }^{\star \star \star} P=2.4 \times 10^{-5}\right.$; FA: $\left.P=0.75\right)$. I Laser-off vs laser-on MI for early component $(P=0.78)$. $\mathbf{m}$ Laser-off vs laseron $\mathrm{Ml}$ for late component $\left({ }^{\star \star \star} P=6.4 \times 10^{-5}\right)$. $\mathbf{n}$ Effect of laser stimulation during No-Go trials on the percentage of trials with licks in the waiting period. $\mathrm{CR}, P=0.14 ; \mathrm{FA},{ }^{\star} P=0.02$. o Effect of laser stimulation on lick rate during waiting period. $\mathrm{FA}, F_{(1,17)}=7.78,{ }^{\star} P=0.01 ; \mathrm{CR}, F_{(1,17)}=1.6, P=0.22$, twoway repeated measures ANOVA. $\mathbf{p}$ Inactivating OFC axons during No-Go trials did not affect hit rate $(P=0.56)$ or $C R$ rate $(P=0.95)$. For $\mathbf{n}-\mathbf{p}, n=$ 18 sessions from 14 mice. Wilcoxon two-sided signed rank test was used for $\mathbf{c}, \mathbf{d}, \mathbf{f}, \mathbf{h}-\mathbf{n}$, and $\mathbf{p}$. For $\mathbf{b}-\mathbf{d}$ and $\mathbf{f}-\mathbf{p}$, source data are provided as a Source Data file. Shadings and error bars, mean \pm s.e.m.

neurons, Wilcoxon signed rank test), resulting in a significant reduction of SI $(P=0.007$, Wilcoxon signed rank test, Fig. $4 \mathrm{f})$. The increase of response to the No-Go stimulus was significant in CR but not in FA trials (CR: $P=1.02 \times 10^{-6}$; FA: $P=0.66, n=$ 169 neurons, Wilcoxon signed rank test, Fig. $4 \mathrm{~h}$ ), leading to a reduction in the amplitude of MI $\left(P=6.4 \times 10^{-4}\right.$, Wilcoxon signed rank test, Fig. 4i). Such effect was observed in both experiments in which laser stimulation was turned on 100-ms before stimulus onset (Supplementary Fig. $6 \mathrm{a}-\mathrm{c}$ ) and at stimulus onset (Supplementary Fig. 6e-g). We also separately analyzed the effect of laser stimulation on the early and late response components. We found that inactivating OFC axons during No-Go trials did not significantly change the firing rates and MI for the early component, but significantly increased firing rates in CR trials and reduced the amplitude of MI for the late component (Fig. $4 \mathrm{j}-\mathrm{m}$ ). As laser stimulation during No-Go trials did not affect the mice's lick rate or orofacial movement during the waiting period in CR trials (Fig. 4n, o, Supplementary Fig. 7 and 
Supplementary Video 1), the laser-induced response increase in CR trials could not be accounted for by a change in movement. Although inactivating OFC axons during No-Go trials significantly influenced the responses of V1 neurons to the No-Go stimulus, it did not change the performance of the mice (Fig. $4 \mathrm{p}$ ). For control mice that GFP was expressed in the OFC, laser stimulation during No-Go trials did not significantly change the responses of V1 neurons to the No-Go stimulus in either CR or FA trials (Supplementary Fig. 6i, j). These data suggest that the OFC top-down projection to V1 contributes to the suppression of late-component $\mathrm{V} 1$ responses to the No-Go stimulus in CR trials.

Response patterns of V1-projecting OFC neurons. Next, we sought to identify V1-projecting OFC neurons with optogenetic tagging method and examined their responses during the $\mathrm{Go} /$ No-Go task. To this end, we first injected rAAV2-retro-hSynChrimsonR-GFP 48 in V1, resulting in the expression of excitatory opsin ChrimsonR in V1-projecting OFC neurons (Supplementary Fig. 8). We performed extracellular recordings from the OFC to monitor the spikes evoked by red laser stimulation (Fig. 5a, b). Using stimulus-associated spike latency test (SALT) for optogenetic identification ${ }^{49}$, we identified V1-projecting OFC neurons as those showing significant laser-evoked responses with short latencies ( $n=22$ out of 1175 units, $P<0.01$, Fig. 5 c, d and Supplementary Fig. 8f). In mice that had been trained for at least 6 days, the responses of identified OFC neurons to the Go and No-Go stimuli were monitored during the behavioral task (Fig. 5e). We defined a response index (RI) as $\left(R_{\text {evoked }}-R_{\text {baseline }}\right) /$ $\left(R_{\text {evoked }}+R_{\text {baseline }}\right)$, where $R_{\text {evoked }}$ and $R_{\text {baseline }}$ represented the firing rates during the waiting period and the baseline period before stimulus onset, respectively. For each neuron, we computed RIs for the responses to the Go stimulus in hit trials and those to the No-Go stimulus in CR (or FA) trials, respectively. We found that the RIs in hit and FA trials were both significantly smaller than zero, whereas those in CR trials were not significantly different from zero (Supplementary Fig. $8 \mathrm{~g}-\mathrm{i}$ ). Thus, the firing rates to the Go stimulus in hit trials and those to the No-Go stimulus in FA trials were reduced as compared to the baseline activity, whereas those to the No-Go stimulus in $\mathrm{CR}$ trials were unchanged. During the behavioral sessions of OFC recordings, the mice showed anticipatory licking during the waiting period for the No-Go stimulus in FA trials as well as for the Go stimulus in hit trials (Supplementary Fig. 8j). These results suggest that V1-projecting OFC neurons reduced firing when the mice expected reward. For V1-projecting OFC neurons, the responses to the No-Go stimulus were significantly lower in FA than in CR trials (Fig. 5f), and MIs were significantly larger than zero $\left(P=6.9 \times 10^{-4}, n=22\right.$ neurons, Wilcoxon signed rank test, Fig. 5g), indicating that their responses exhibit outcomeexpectancy modulation opposite to that found for V1 neurons. The spike rate of V1-projecting OFC neurons was not correlated with the lick rate (Supplementary Fig. 8k), and the histogram of lick-triggered spikes did not show increase in response following lick (Supplementary Fig. 81, m). For those trials in which no lick occurred within the first $0.5 \mathrm{~s}$ following stimulus onset, the MIs of V1-projecting OFC neurons remained significantly larger than zero (Supplementary Fig. 8n). Thus, the response difference between CR and FA trials for these OFC neurons could not be attributed to licking. Given that activating OFC axons in V1 caused response suppression of V1 neurons (Fig. 1), the decreased activity of V1-projecting OFC neurons in FA, but not CR, trials (Fig. 5f, g) may provide an explanation of the observation that $\mathrm{V} 1$ responses to the No-Go stimulus were higher in FA trials (Fig. 3k, 1).
OFC projection to $\mathrm{V} 1$ contributes to learning. The above results showed that, although inactivating OFC projection to V1 could affect $\mathrm{V} 1$ responses, it did not change the behavioral performance (Fig. 4p). As these mice had been trained before the optogenetic perturbation, we next examined whether perturbation of OFC projection from the first day of training influences the learning process. To inactivate the OFC projection to V1, we expressed Jaws in the OFC (Fig. 6a and Supplementary Fig. 9). For control mice, EGFP alone was expressed in the OFC (Fig. 6a). Both the Jaws-expressing and the EGFP-expressing mice were divided into two groups. The laser stimulation was applied to V1 during No-Go trials for one group (Fig. 6b and Supplementary Fig. 9) and during Go trials for another group (Fig. 6c and Supplementary Fig. 9). Throughout the learning process, each session consisted of interleaved blocks of laser-on and laser-off trials (20 trials/block).

For the group of Jaws-expressing mice with laser stimulation during No-Go trials, the behavioral performance were similar between laser-on and laser-off trials (Supplementary Fig. 9e), indicating that inactivation of the OFC axons in V1 during No-Go trials did not affect the immediate performance and corroborating the result in Fig. 4p. However, compared to the EGFP-expressing control mice, laser stimulation during No-Go trials slowed the learning in Jaws-expressing mice (Fig. 6b). For the experiments that laser stimulation was applied during Go trials, the performance was higher in laser-on than in laser-off trials for both the control and Jaws-expressing mice (Supplementary Fig. 9g, h), which may be due to the possibility that laser stimulation served as a cue to guide the mice's behavior. Nevertheless, the Jaws-expressing and control mice did not differ in their learning curves (Fig. 6c), indicating that inactivating OFC axons in V1 during Go trials had no effect on learning. Together, the results demonstrate that learning to correctly reject the reward-irrelevant No-Go stimulus requires the activity of OFC projection to $\mathrm{V} 1$.

We further examined whether the learning process could be affected by optogenetic activation of the OFC top-down projection. We found that activation of OFC axons in V1 during No-Go or Go trials both caused an increase in behavioral performance in the session with laser stimulation, but the effect disappeared in the next session without laser stimulation (Supplementary Fig. 10). Such transient effect was likely due to antidromic activation of OFC neurons (Fig. 1g) that may activate other neurons or pathways involved in performing the task. Given that SST interneurons in V1 were innervated by OFC axons (Supplementary Fig. 2) and were the predominant interneuron subtype showing activity elevation following photostimulation of OFC neurons in vivo (Fig. 2i, j), we next examined the activity of SST interneurons in the Go/No-Go task and whether activating SST interneurons in V1 could affect learning. We used fiber photometry to measure the activity of SST interneurons by injecting AAV2/9-hSyn-FLEX-GCaMP6s-WPRE in V1 of SST-Cre mice (Supplementary Fig. 11a). In mice that had been trained for at least 4 days, the responses of SST interneurons to the No-Go stimulus during the waiting period were significantly higher in CR than in FA trials (Supplementary Fig. 11b, c). This response modulation was consistent with that found for the V1-projecting OFC neurons and may be partly attributed to the OFC projection to V1. To examine the role of SST interneurons in the learning of Go/No-Go task, we used SST-Cre mice in which AAVs encoding Cre-dependent ChrimsonR or tdTomato alone were injected into V1 (Fig. 6d). In each session of the learning process, laser stimulation of V1 was applied during No-Go trials, and blocks of laser-on and laser-off trials were interleaved. We found that activating SST interneurons in V1 during No-Go trials did not affect immediate performance 
a
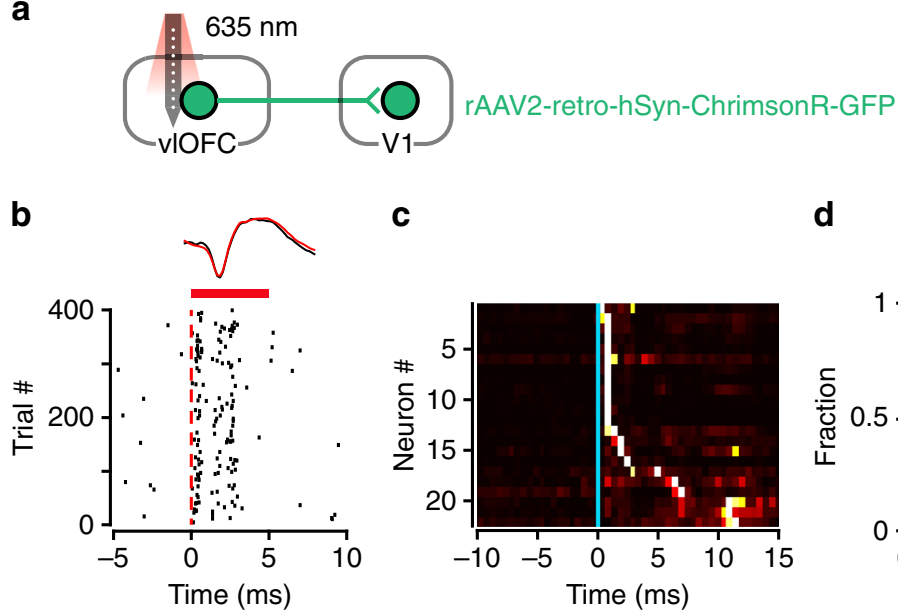

C

d
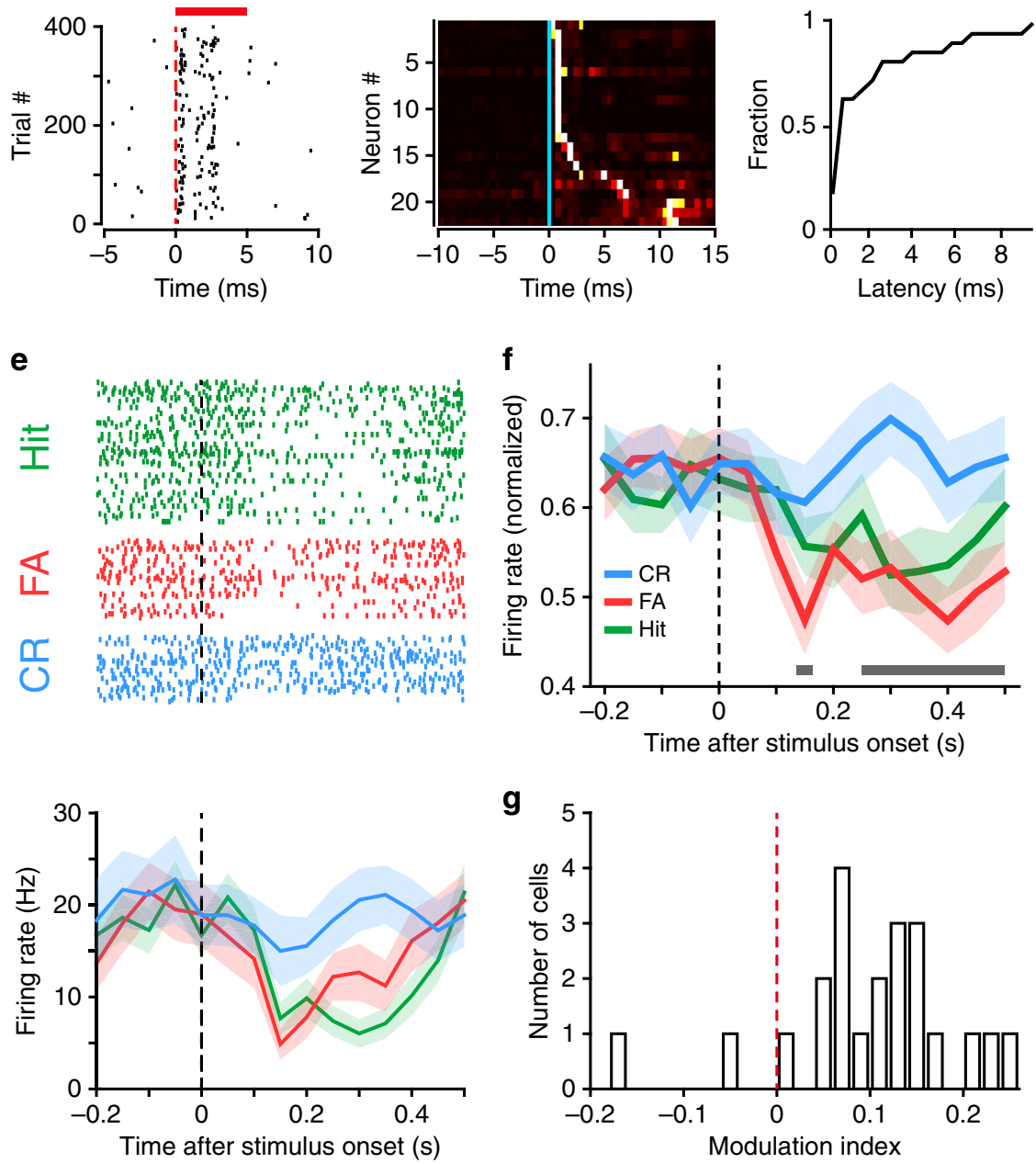

Fig. 5 V1-projecting OFC neurons reduce activity during reward expectation. a Schematic of the strategy for phototagging V1-projecting OFC neurons. b Spike raster of an identified V1-projecting OFC neuron aligned to laser onset. Red bar, duration of laser stimulation. Top, mean waveforms for spontaneous (black) and laser-evoked (red) spikes. c PSTHs aligned to laser onset (blue line) for all identified neurons. The responses were normalized by peak value and sorted by peak latency. Colors from black to white indicate increase in firing rate. $\mathbf{d}$ Cumulative distribution of laser-evoked spike latency for all identified neurons. e Spike rasters and PSTHs of an identified V1-projecting OFC neuron during hit (green), FA (red) and CR (blue) trials, aligned to stimulus onset. $\mathbf{f}$ The firing rates of each neuron were normalized by the maximum of the peak values in hit, FA, and CR trials, and were averaged across neurons. Horizontal bar, timepoints with $P<0.05$, two-way repeated measures ANOVA $F_{(1,21)}=17.86, P=3.79 \times 10^{-4}$ followed by Sidak's multiple comparisons test. $\mathbf{g}$ Distribution of MIs for identified V1-projecting OFC neurons. $P=6.9 \times 10^{-4}, n=22$ neurons, Wilcoxon two-sided signed rank test. For $\mathbf{c}-\mathbf{g}$, source data are provided as a Source Data file. Shadings, mean \pm s.e.m.

in each session, as shown by similar d' and CR rate between laseron and laser-off trials (Supplementary Fig. 9j). However, the d' and CR rate of ChrimsonR-expressing mice were significantly higher than those of control mice expressing tdTomato only (Fig. 6e). After multiple sessions with laser stimulation during No-Go trials, the higher d' and CR rate in ChrimsonR-expressing mice persisted on later sessions where laser stimulation was no longer applied (Fig. 6e), indicating that the improved performance was indeed due to learning. Thus, SST interneurons in V1 play an important role in the learning of correct rejection for the No-Go stimulus, serving as a substrate for modulating visual associative learning by the OFC to $\mathrm{V} 1$ projection.

\section{Discussion}

In this study, we demonstrated that activation of OFC projection to $\mathrm{V} 1$ caused reduction of visual responses in V1 by preferentially recruiting SST interneurons in the local circuit. In mice performing a Go/No-Go visual task, the activity of OFC projection to V1 played an essential role in the outcome-expectancy modulation of V1 responses to the reward-irrelevant No-Go stimulus. Phototagging experiments showed that the responses of V1projecting OFC neurons to the No-Go stimulus were decreased when the mice incorrectly expected reward. Importantly, we revealed that the OFC projection to $\mathrm{V} 1$ is critical for the learning of correctly rejecting the No-Go stimulus. 
a

AAV-CaMKIl $\alpha$-Jaws-GFP

or

AAV-CaMKII $\alpha$-EGFP

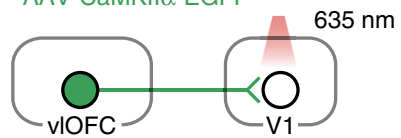

b

$$
\text { Laser stimulation during No-Go trials }
$$
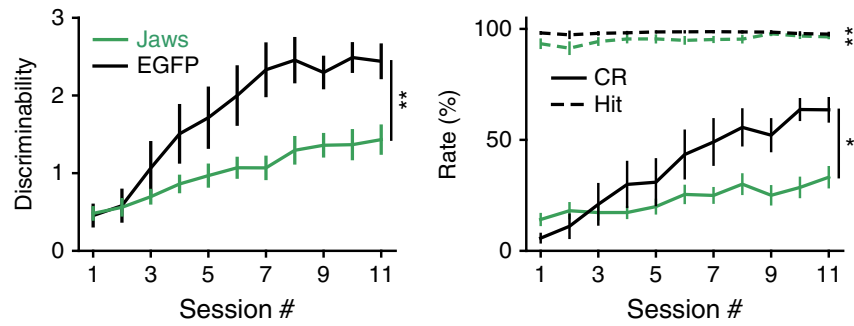

d

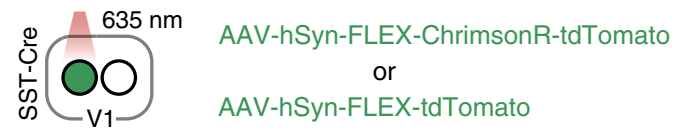

C Laser stimulation during Go trials
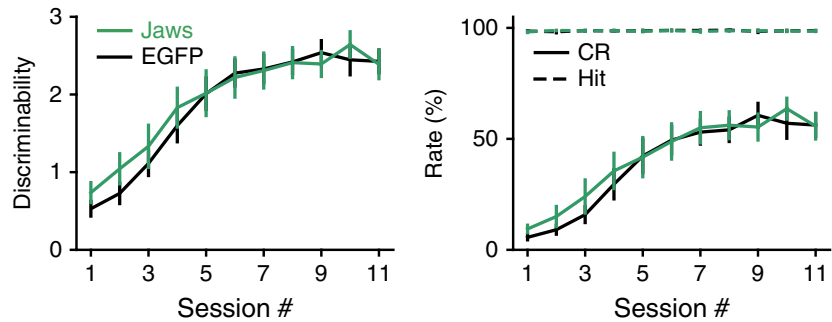

e

Laser stimulation during No-Go trials
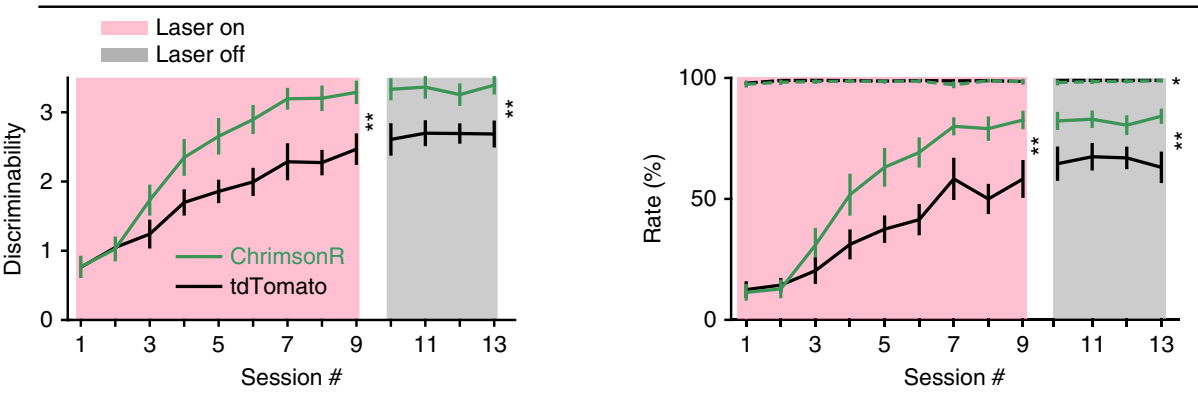

Fig. 6 OFC projection to V1 contributes to visual associative learning. a Schematic of the strategy for inactivating OFC projection to V1. $\mathbf{b}$ Effect of inactivating OFC projection to V1 during No-Go trials. $n=9$ and 13 for EGFP- and Jaws-expressing mice, respectively. For discriminability: $F_{(1,20)}=10.24$, ${ }^{\star \star} P=0.004$. For hit rate: $F_{(1,20)}=13.38,{ }^{\star \star} P=0.002$. For $C R$ rate: $F_{(1,20)}=6.69,{ }^{\star} P=0.02$. Two-way ANOVA with mixed design. $\mathbf{c}$ Effect of inactivating OFC projection to V1 during Go trials. $n=11$ and 12 for EGFP- and Jaws-expressing mice, respectively. For discriminability: $F_{(1,21)}=0.13, P=0.72$. For hit rate: $F_{(1,21)}=0.24, P=0.63$. For $C R$ rate: $F_{(1,21)}=0.13, P=0.72$. Two-way ANOVA with mixed design. $\mathbf{d}$ Schematic of the strategy for activating SST interneurons in V1. e Effect of activating SST interneurons in V1 during No-Go trials. $n=10$ and 11 for tdTomato- and ChrimsonR-expressing mice, respectively. Sessions 1-9, with laser stimulation; sessions 10-13, without laser stimulation. For discriminability in sessions 1-9: $F_{(1,19)}=10.44$, ${ }^{\star \star} P=0.004$; discriminability in sessions 10-13: $F_{(1,19)}=11.93,{ }^{\star \star} P=0.003$. For hit rate in sessions 1-9: $F_{(1,19)}=4.37, P=0.05$; hit rate in sessions 10-13: $F_{(1,19)}=6.07$, ${ }^{\star} P=0.02$. For $C R$ rate in sessions 1-9: $F_{(1,19)}=10.63,{ }^{\star} P=0.004 ; C R$ rate in sessions 10-13: $F_{(1,19)}=9.84,{ }^{\star \star} P=0.005$. Two-way ANOVA with mixed design. For b, c, e, source data are provided as a Source Data file. Error bars, mean \pm s.e.m.

Top-down projections to primary sensory areas play an important role in sensory processing and sensory-guided behavior ${ }^{35,50}$. Activating the projections from cingulate region of the frontal cortex to V1 enhances and suppresses V1 responses for the sites near and surround axonal activation, respectively, consistent with the effect of top-down modulation in selective attention $^{36}$. Top-down projections from the anterior cingulate cortex to V1 carried stimulus prediction, which could be used to compute deviations from expectations and guide learning 39 . Associative learning also enhances the effect of top-down inputs from the retrosplenial cortex in modulating V1 responses ${ }^{42}$. In our study, we found that the responses of V1 neurons to the reward-irrelevant No-Go stimulus were modulated by outcome expectancy, being lower when the mice' outcome expectation was correct than when it was wrong. Similar to that found in the somatosensory cortex ${ }^{51,52}$, the modulation of V1 responses by outcome expectation was evident in the late response component after stimulus onset. The response modulation in the late response component was reduced by optogenetic inactivation of OFC projection to $\mathrm{V} 1$, consistent with the role of OFC in the encoding of outcome expectation ${ }^{2}$. Chronic inactivation of OFC projection to V1 during No-Go trials slowed the improvement of CR rate, without affecting the performance per se, suggesting that the OFC top-down modulation of V1 responses to the No-Go stimulus is important for learning.

Few studies have recorded from behaving animals the responses of higher cortical neurons that provide top-down projections to $\mathrm{V}^{53}$, and examined the causal role of top-down inputs to V1 in learning ${ }^{54}$. The CTB and virus retrograde tracing in our study showed that, the OFC neurons sending projections to V1 were in the ventrolateral OFC. The V1-projecting OFC neurons target all three subtypes of inhibitory interneurons in V1, similar to that found for OFC projections to auditory cortex ${ }^{37}$ and cingulate cortex projections to $\mathrm{V}^{36}$. We found that optogenetic stimulation of OFC neurons in vivo preferentially activated SST interneurons in V1. To study the functional role of 
OFC projection to $\mathrm{V} 1$, we used a Go/No-Go visual task, in which the learning depended on the improvement of correct rejection for the reward-irrelevant No-Go stimulus. Many studies showed that the OFC neurons increased firing in anticipation of reward ${ }^{2}$, while some studies also found that a fraction of OFC neurons decreased firing to reward-predicting cues ${ }^{27,31,55}$. We found that, those OFC neurons not identified as V1-projecting showed higher firing rates to the No-Go stimulus in trials when mice incorrectly expected reward as well as to the Go stimulus when the reward expectation was correct (Supplementary Fig. 8o). By contrast, the V1-projecting OFC neurons exhibited response decrease relative to the baseline when the mouse expected reward for the Go stimulus in hit trials or for the No-Go stimulus in FA trials. This response decrease may account for the observation that inactivation of OFC projection to V1 during Go trials did not affect V1 responses to the Go stimulus or inactivation during No-Go trials did not affect responses to the No-Go stimulus in FA trials. For the No-Go stimulus, the responses of V1-projecting OFC neurons in CR trials were not different from the baseline response, and thus were higher than those in FA trials. Because the learning of Go/No-Go visual task manifested as an increase in the CR rate, the responses of V1-projecting OFC neurons to the No-Go stimulus likely increased with training as the percentage of CR trials increased. During No-Go trials, OFC projection to V1 may cause stronger activation of local SST interneurons in CR than in FA trials, leading to lower $\mathrm{V} 1$ responses in $\mathrm{CR}$ than in FA trials. Indeed, we found that the responses of SST interneurons in V1 to the No-Go stimulus were higher in CR than in FA trials, and inactivating OFC top-down projection increased $\mathrm{V} 1$ responses to the No-Go stimulus in CR trials. As the percentage of CR trials increased with training, the responses of $\mathrm{V} 1$ neurons to the No-Go stimulus likely decreased, representing a change in stimulus salience. Chronic activation of SST interneurons in V1 during No-Go trials facilitated learning, suggesting that recruitment of local SST interneurons by OFC top-down projection may promote learning by reducing V1 responses to reward-irrelevant stimulus. This result is also in line with recent reports that the activity of SST interneurons during learning may reflect the signals from long-range inputs ${ }^{42,56}$.

On the other hand, we found that optogenetic inactivation of OFC axons in V1 did not affect the immediate performance of mice. One possible explanation is that during the waiting period, the mice's decision was determined by the responses of the OFC neurons, which could be seen from the divergence of response curves between FA and CR trials (Fig. 5f and Supplementary Fig. 8o), so that modulation of V1 responses would not affect the mice's performance in current trial. A second possibility is that the effect of response modification in V1 needs to accumulate over multiple sessions to impact behavioral performance. As the OFC receives inputs from visual cortex ${ }^{32-34}$, the response modification in V1 may also in turn contribute to the update of outcome expectation signal in the OFC, potentially contributing to change of performance in future trials.

Studies using outcome devaluation and Pavlovian overexpectation tasks have revealed that the OFC is necessary for using expectations of specific outcome to guide behavior and learning 3,57 . The outcome predictions signaled by the OFC neurons could be utilized by downstream regions such as ventral striatum $^{58,59}$, dorsal striatum ${ }^{58,60}, \mathrm{BLA}^{61}$, and $\mathrm{VTA}^{23,29,62}$. OFC lesion or inactivation disrupted the prediction errors signaled by dopaminergic neurons ${ }^{29,62}$ and expected reward values by putative non-dopaminergic neurons ${ }^{62}$ in VTA. While optogenetic inhibition of VTA-projecting OFC neurons did not impair the acquisition of Pavlovian trace conditioning, it impaired extinction learning and memory ${ }^{27}$. In addition, distinct OFC circuits were found to mediate different aspects of reinforcement learning in value-based decision making ${ }^{63}$. We found that the outcome expectation signals of $\mathrm{OFC}$ neurons are sent to $\mathrm{V} 1$ to regulate visual associative learning. As the OFC projects to other sensory cortices in addition to visual cortex ${ }^{32}$, the OFC top-down projection may also play an important role in associative learning for other sensory modalities.

\section{Methods}

Animals. All animal procedures were approved by the Animal Care and Use Committee at the Institute of Neuroscience, Chinese Academy of Sciences, and were in accordance with the guidelines of the Animal Advisory Committee at the Shanghai Institutes for Biological Sciences.

We used the following mice: GAD67-GFP (CB6-Tg(Gad1-EGFP)G42Zjh), CaMKIIa-Cre (B6.Cg-Tg(Camk2a-cre)T29-1Stl), SST-Cre (Sst ${ }^{\text {tm2.1(cre)Zjh }), ~ P V-C r e ~}$ (B6;129P2-Pvalb ${ }^{\text {tm1(cre)Arbr }), ~ V I P-C r e ~(V i p ~}{ }^{\text {tml(cre)Zjh }), ~ S S T:: A i 9 ~(g e n e r a t e d ~ b y ~}$

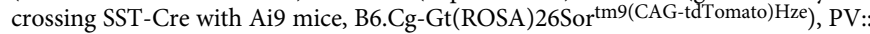
Ai9, VIP::Ai9 and C57BL/6 mice. Adult (2-4 months) male mice were used for all experiments. Mice were housed under standardized conditions with a $12 \mathrm{~h}: 12 \mathrm{~h}$ light/dark cycle, with the temperature controlled at $22-23{ }^{\circ} \mathrm{C}$ and humidity at $40-70 \%$.

Adeno-associated Virus (AAVs). We used the following AAVs: rAAV2-retrohSyn-Cre (titer: $3.2 \times 10^{12}$ viral particles $\mathrm{ml}^{-1}$ ) and AAV2/8-EF1a-DIO-EYFPWPRE (titer: $6.9 \times 10^{12}$ viral particles $\mathrm{ml}^{-1}$, for visualizing OFC axons in V1 in Fig. 1b); AAV2/8-CaMKIIa-hChR2(H134R)-mCherry (titer: $8.26 \times 10^{12}$ viral particles $\mathrm{ml}^{-1}$ ) and AAV2/8-hSyn-ChrimsonR-GFP (titer: $6.58 \times 10^{12}$ viral particles $\mathrm{ml}^{-1}$ ) (for activating OFC projection to V1 in Fig. 1, Supplementary Fig. 1 and Supplementary Fig. 10, or for activating OFC in Fig. $2 \mathrm{~g}-\mathrm{k}$ ); AAV2/8-CaMKIIahChR2(H134R)-EYFP (titer: $8.66 \times 10^{12}$ viral particles $\mathrm{ml}^{-1}$; for activating OFC projection to V1 in Fig. $2 \mathrm{a}-\mathrm{f}$ ); AAV2/9-hSyn-FLEX-GCaMP6s-WPRE (titer: $6.9 \times$ $10^{12}$ viral particles $\mathrm{ml}^{-1}$; for fiber photometry experiment in Fig. $2 \mathrm{~g}-\mathrm{k}$ and Supplementary Fig. 11); AAV2/9-EF1a-DIO-His-EGFP-2A-TVA-WPRE (titer: $1.26 \times$ $10^{12}$ viral particles $\mathrm{ml}^{-1}$ ), AAV2/9-EF1 $\alpha$-DIO-RVG-WPRE (titer: $3.12 \times 10^{12}$ viral particles $\mathrm{ml}^{-1}$ ) and RV-EnvA- $\Delta$ G-dsRed (titer: $1 \times 10^{8}$ viral particles $\left.\mathrm{ml}^{-1}\right)($ for retrograde monosynaptic tracing in Supplementary Fig. 2); AAV2/8-hSyn-JawsKGC-GFP-ER2 (titer: $5.4 \times 10^{12}$ viral particles $\mathrm{ml}^{-1}$; for inactivating OFC projection to V1 in Fig. 4, Supplementary Fig. 1, Supplementary Fig. 6 and Supplementary Fig. 7); rAAV2-retro-hSyn-ChrimsonR-GFP (titer: $5.63 \times 10^{12}$ viral particles $\mathrm{ml}^{-1}$; for optogenetic tagging of V1-projecting OFC neurons in Fig. 5 and Supplementary Fig. 8); AAV2/8-CaMKII $\alpha$-Jaws-KGC-GFP-ER2 (titer: $5.38 \times 10^{12}$ viral particles $\mathrm{ml}^{-1}$; for inactivating OFC projection to V1 in Fig. 6 and Supplementary Fig. 9); AAV2/8-hSyn-FLEX-ChrimsonR-tdTomato (titer: $3.7 \times 10^{12}$ viral particles $\mathrm{ml}^{-1}$; for activating SST interneurons in Fig. 6 and Supplementary Fig. 9); AAV2/8-CaMKIIa-EGFP-WPRE (titer: $5.8 \times 10^{12}$ viral particles $\mathrm{ml}^{-1}$ ), AAV2/8CaMKIIa-mCherry (titer: $5.7 \times 10^{12}$ viral particles $\mathrm{ml}^{-1}$ ), AAV2/8-hSyn-FLEXtdTomato (titer: $5.1 \times 10^{12}$ viral particles $\mathrm{ml}^{-1}$ ) and AAV-hSyn-EGFP-WPRE (titer: $7.3 \times 10^{12}$ viral particles $\mathrm{ml}^{-1}$ ) (for optogenetic or fiber photometry experiments as a control group in Fig. 2i, Fig. 6, Supplementary Fig. 1, Supplementary Fig. 6 and Supplementary Fig. 9).

Surgery. The mice were anesthetized with a mixture of midazolam $\left(5 \mathrm{mg} \mathrm{kg}^{-1}\right)$, fentanyl $\left(0.05 \mathrm{mg} \mathrm{kg}^{-1}\right)$ and medetomidine $\left(0.5 \mathrm{mg} \mathrm{kg}^{-1}\right)$, and were head-fixed in a stereotaxic apparatus. For behavioral experiments without optogenetic manipulation, head plates were implanted before behavioral training. For in vivo recording and behavioral experiments with optogenetic manipulation, head plates were implanted after the virus injection. The virus was injected with a glass pipette (10-20 $\mu \mathrm{m}$ tip diameter) using a syringe pump (Harvard Apparatus). To observe the OFC axons in V1, we injected rAAV2-retro-hSyn-Cre $(100 \mathrm{nl})$ in V1 (AP, $-3.5 \mathrm{~mm}$; ML, $2.4 \mathrm{~mm}$; DV, $0.5 \mathrm{~mm}$ ) and AAV2/8-EF1a-DIO-EYFP-WPRE (500 $\mathrm{nl}$ ) in the OFC (AP, $2.7 \mathrm{~mm}$; ML, $0.88 \mathrm{~mm}$; DV, $1.8 \mathrm{~mm}$ ). To manipulate the OFC to $\mathrm{V} 1$ projection in $\mathrm{C} 57 \mathrm{BL} / 6$ mice, a craniotomy was made above the right $\mathrm{OFC}$ (AP, $2.7 \mathrm{~mm}$; ML, $0.88 \mathrm{~mm}$ ), and $500 \mathrm{nl}$ of AAV (AAV2/8-hSyn-Jaws-KGC-GFPER2, AAV2/8-CaMKIIa-Jaws-KGC-GFP-ER2, AAV2/8-CaMKIIa-hChR2

(H134R)-mCherry, AAV2/8-CaMKIIa-hChR2(H134R)-EYFP, AAV2/8-hSynChrimsonR-GFP; or the control virus: AAV2/8-CaMKIIa-EGFP-WPRE or AAV2/ 8-CaMKIIa-mCherry) were injected into the cortex at a depth of $1.8 \mathrm{~mm}$. A rectangular region on the skull above $\mathrm{V} 1$ ( $\mathrm{AP},-3.2$ to $-3.8 \mathrm{~mm}$; ML, 2.0 to $2.8 \mathrm{~mm}$ ) ipsilateral to the OFC injection site was marked by cutting and permanent red ink. The marked skull region above V1 was covered with tissue glue (Vetbond, $3 \mathrm{M}$ ) until optogenetic manipulation or in vivo recording. For the experiments to block antidromic spiking of OFC neurons caused by laser stimulation of OFC axons in $\mathrm{V} 1$, a cannula $(0.41 \mathrm{~mm}$ diameter) was implanted $500 \mu \mathrm{m}$ above the virus injection site in the OFC. For fiber photometry recording of OFC stimulation-evoked activity in V1, AAV2/8-CaMKIIa-hChR2(H134R)-mCherry (600 nl) was injected to the right OFC at a depth of $1.8 \mathrm{~mm}$ and AAV2/9-hSyn-FLEX-GCaMP6s-WPRE (300 $\mathrm{nl}$ ) was injected to the right V1 at a depth of $0.5 \mathrm{~mm}$ in SST-Cre, PV-Cre, or VIPCre mice. Following the virus injection, one optical fiber $(200 \mu \mathrm{m}$ diameter, NA 0.37) was inserted $100 \mu \mathrm{m}$ above the injection site in the OFC and another one 
touching the dura of injection site in V1. For fiber photometry recording of SST interneurons in V1 in behaving mice, AAV2/9-hSyn-FLEX-GCaMP6s-WPRE (300 $\mathrm{nl}$ ) was injected to the right V1 at a depth of $0.5 \mathrm{~mm}$ in SST-Cre mice, and optical fiber $(200 \mu \mathrm{m}$ diameter, NA 0.37$)$ was inserted to touch the dura of V1. For phototagging of V1-projecting OFC neurons, a craniotomy was made above the right V1 (AP, $-3.5 \mathrm{~mm}$; ML, $2.4 \mathrm{~mm}$ ), and rAAV2-retro-hSyn-ChrimsonR-GFP $(300 \mathrm{nl})$ were injected into the cortex at a depth of $0.5 \mathrm{~mm}$. To activate SST interneurons in V1 of SST-Cre mice, AAV2/8-hSyn-FLEX-ChrimsonR-tdTomato $(300 \mathrm{nl})$ were injected into $\mathrm{V} 1$ at a depth of $0.5 \mathrm{~mm}$, and the craniotomy was protected with a silicone elastomer (Kwik-Sil, WPI). After the virus injection, a stainless-steel headplate was fixed to the skull using dental cement. For mice used for Go/No-Go behavior with optogenetic manipulation of OFC axons in V1 or SST interneurons in V1, dental cement mixed with $50 \%$ carbon powder was used to cover the skull except the region above V1. The mice were injected with carprofen $\left(5 \mathrm{mg} \mathrm{kg}^{-1}\right)$ subcutaneously after the surgery for 3 days, and were allowed to recover with food and water ad libitum for at least 7 days.

For infusion of tetrodotoxin (TTX) in the OFC, $1 \mu$ lof TTX $(4 \mu \mathrm{M})$ was injected into the OFC through the implanted cannula $0.5-1 \mathrm{~h}$ before the in vivo recording.

Fluorescently conjugated Cholera toxin subunit B (CTB-555, $2 \mu \mathrm{g} \mu \mathrm{l}^{-1}, 300 \mathrm{nl}$, Invitrogen) was injected unilaterally into $\mathrm{V} 1 \mathrm{AP},-3.5 \mathrm{~mm}$; $\mathrm{ML}, 2.4 \mathrm{~mm}$ ) in C57BL/6 or GAD67-GFP mice. In some C57BL/6 mice, both CTB and rAAV2retro-hSyn-ChrimsonR-GFP were injected in V1 to estimate the percentage of OFC neurons co-labeled by GFP and CTB. The histology experiments were performed 2 weeks after the injection.

Glycoprotein-deleted $(\Delta \mathrm{G})$ and EnvA-pseudotyped rabies virus (RV-EnvA- $\Delta \mathrm{G}$ dsRed) was used for retrograde monosynaptic tracing from different types of V1 neurons ${ }^{43}$. TVA receptor and rabies glycoprotein were expressed in Cre-positive neurons by co-injection of AAV2/9-EF1 $\alpha$-DIO-His-EGFP-2A-TVA-WPRE and AAV2/9-EF1 $\alpha$-DIO-RVG-WPRE (300 nl) in V1 in CaMKIIa-Cre, PV-Cre, SSTCre and VIP-Cre mice. RV-EnvA- $\Delta$ G-dsRed $(300 \mathrm{nl})$ was injected in the same site two weeks later. The histology experiments were performed 8 days after the RV injection.

In vivo extracellular recording. Recordings with optogenetic stimulation were performed at least 3 weeks after the virus injection. For anesthetized experiments, mice were injected with chlorprothixene $\left(3.2 \mathrm{mg} \mathrm{kg}^{-1}\right)$ subcutaneously and anesthetized with urethane $\left(0.7-1.0 \mathrm{~g} \mathrm{~kg}^{-1}\right)$ intraperitoneally. The mouse was headfixed in a stereotaxic frame and its body temperature was maintained at $37^{\circ} \mathrm{C}$ through a heating blanket (FHC Inc.). A craniotomy ( 1 mm diameter) was made above V1 (AP, $-3.5 \mathrm{~mm}$; ML, $2.4 \mathrm{~mm}$ ). For recordings in awake mice, the body of the mouse was restricted in a circular plastic tube and the headplate was fixed to a holder attached to the stereotaxic apparatus. While the animal was anesthetized with isoflurane (1-2\%), a craniotomy ( 1 mm diameter) was made above V1 (AP, $-3.5 \mathrm{~mm} ; \mathrm{ML}, 2.4 \mathrm{~mm}$ ) or OFC (AP, $2.7 \mathrm{~mm}$; ML $0.88 \mathrm{~mm}$ ). The dura was removed, and the craniotomy was covered by $\sim 1 \%$ agarose dissolved in artificial cerebral spinal fluid (ACSF) and protected by a silicone elastomer (Kwik-Cast, WPI). The mouse was allowed to recover from the anesthesia in home cage for at least $1 \mathrm{~h}$. The recordings were made with multi-site silicon probes $(\mathrm{A} 1 \times 16-3 \mathrm{~mm}-$ 50-177 or A1 ×16-5mm-50-177, NeuroNexus Technologies; ASSY-77.2-64-6, Diagnostic Biochips, Inc.) mounted on a manipulator (MP-225, Sutter Instrument Company). For some recordings, the silicon probe was coated with DiO (Invitrogen) to allow post hoc recovery of penetration track. After finishing the recordings from awake mice, the electrode was retracted. The craniotomy was cleaned with ACSF, covered with $\sim 1 \%$ agarose and protected with a silicone elastomer (Kwik-Cast, WPI). After the experiments, the mouse was euthanized by an overdose of sodium pentobarbital.

The neural responses were amplified and filtered using a Cerebus 64-channel system (Blackrock microsystems). Local field potential signals were sampled at 2 $\mathrm{kHz}$ with a wide-band front-end filter $(0.3-500 \mathrm{~Hz})$. Spiking signals were sampled at $30 \mathrm{kHz}$. To detect the waveforms of spikes, we bandpass filtered the signals at $250-7500 \mathrm{~Hz}$ and set a threshold at 3.5 s.d. of the background noise. Spikes were sorted offline using the Offline Sorter (Plexon Inc.) based on cluster analysis of principle component amplitude. Spike clusters were considered to be single units if the percentage of spikes with interspike interval $<1 \mathrm{~ms}$ was lower than $0.3 \%$ and the $P$-value for multivariate analysis of variance tests on clusters was less than 0.05 .

Slice preparation and recording. We used C57BL/6, SST::Ai9, PV::Ai9, and VIP:: Ai9 mice for slice recordings. Mice that had been injected with AAV2/8-CaMKIIahChR2(H134R)-EYFP in the OFC were anesthetized with isoflurane and perfused with ice-cold cutting solution containing the following (in $\mathrm{mM}$ ): sucrose $234, \mathrm{KCl}$ 2.5, $\mathrm{NaH}_{2} \mathrm{PO}_{4} 1.25, \mathrm{MgSO}_{4} 10, \mathrm{CaCl}_{2} 0.5, \mathrm{NaHCO}_{3} 26$ and glucose 11 (300-305 mOsm). The mouse brain was dissected, and coronal slices $(300 \mu \mathrm{m})$ were prepared using a vibratome (Leica VT1200S) in the ice-cold cutting solution. The prepared brain slices of $\mathrm{V} 1$ were incubated in ACSF containing the following (in $\mathrm{mM}$ ): $\mathrm{NaCl}$ $126, \mathrm{KCl} 2.5, \mathrm{NaH}_{2} \mathrm{PO}_{4} 1.25, \mathrm{MgCl}_{2} 2, \mathrm{CaCl}_{2} 2, \mathrm{NaHCO}_{3} 26$ and glucose 10 (300-305 mOsm) for $30-45 \mathrm{~min}$ at $34^{\circ} \mathrm{C}$, and then kept at room temperature. The cutting solution and ACSF were bubbled with $95 \% \mathrm{O}_{2}$ and $5 \% \mathrm{CO}_{2}$.

Whole-cell recordings of layer $2 / 3 \mathrm{~V} 1$ neurons in voltage-clamp mode were made at room temperatures $\left(25-28^{\circ} \mathrm{C}\right)$ with a Multiclamp $700 \mathrm{~B}$ amplifier and a Digidata 1440A (Molecular Devices). The electrodes were filled with a Cs-based low $\mathrm{Cl}^{-}$internal solution containing the following (in $\mathrm{mM}$ ): $\mathrm{CsMeSO}_{3} 130, \mathrm{MgCl}_{2}$ 1, $\mathrm{CaCl}_{2}$ 1, HEPES 10, QX-314 2, EGTA 11, Mg-ATP 2, Na-GTP 0.3 (pH 7.3, 295 $\mathrm{mOsm})$. Excitatory and inhibitory currents were recorded at $-70 \mathrm{mV}$ and $0 \mathrm{mV}$, respectively. Different types of V1 inhibitory neurons were identified by tdTomatoexpressing neurons in SST::Ai9, PV::Ai9 and VIP::Ai9 mice. Pyramidal neurons were identified based on the morphology of tdTomato-negative cells and verified by staining of biocytin, which was included in the internal solution. Picrotoxin $(50$ $\mu \mathrm{M}$, Tocris) and $\mathrm{NBQX}\left(10 \mu \mathrm{M}\right.$, Tocris) were used to block $\mathrm{GABA}_{\mathrm{A}}$ receptor and AMPA receptor mediated currents, respectively. Data were sampled at 10 or 20 $\mathrm{kHz}$ and analyzed with pCLAMP 10 (Molecular Devices). To determine whether the laser stimulation-evoked EPSC is significant, we computed the baseline by averaging the responses within $10 \mathrm{~ms}$ before laser onset, and compared the peak amplitude of EPSC with the baseline using Wilcoxon signed rank test.

Fiber photometry. For fluorescence $\mathrm{Ca}^{2+}$ recordings, light from a 473-nm LED was reflected by a dichroic mirror (MD498, Thorlabs). The emission signals collected through the implanted optical fiber in V1 were filtered by a bandpass filter (MF525-39, Thorlabs) and detected by a photomultiplier tube (PMT, R3896, Hamamatsu). The light at the tip of the optical fiber was adjusted to $10-30 \mu \mathrm{W}$ to minimize bleaching. An amplifier converted the output of the PMT to voltage signals, which were digitized using a data acquisition card (USB6009, National Instrument) at $200 \mathrm{~Hz}$ with custom-written programs.

Visual stimulation. For in vivo recording experiments, visual stimuli were presented on a 17" LCD monitor (Dell P170S, mean luminance of $35 \mathrm{~cd} \mathrm{~m}^{-2}$, refresh rate $60 \mathrm{~Hz}$ ) placed $9 \mathrm{~cm}$ away from the eye contralateral to the recording site, subtending $112.6^{\circ} \times 124.2^{\circ}$ of visual space. Gamma correction was used to calibrate the monitor. The position of the monitor was adjusted such that the receptive fields (RFs) of the recorded neurons were at the center of the monitor. To locate the RFs of V1 neurons, we presented sparse noise stimuli over a black background, in which a white square $\left(21^{\circ} \times 21^{\circ}\right)$ was flashed for $33.3 \mathrm{~ms}$ on a $112.6^{\circ}$ square grid in a pseudorandom sequence (100 repeats). To measure orientation tuning with and without inactivating OFC axons in V1, we presented drifting gratings $\left(96^{\circ} \times 96^{\circ}\right.$, spatial frequency $=0.03$ cycles $\mathrm{deg}^{-1}$, temporal frequency $=2 \mathrm{~Hz}$, contrast $=$ $100 \%$ ) at 12 different directions (spaced at $30^{\circ}$ ) in a random sequence. Each stimulus was repeated 14 times for both laser-off and laser-on conditions. Each trial of the stimulus started with $1 \mathrm{~s}$ of gray screen, followed by $0.5 \mathrm{~s}$ of the first frame of grating and $2 \mathrm{~s}$ of the drifting grating.

For behavioral experiments, oriented gratings $\left(90^{\circ} \times 90^{\circ}\right.$, spatial frequency $=$ 0.04 cycles $/$ deg, contrast $=100 \%$ ) were presented on a 17 " LCD monitor (Dell E1713S, mean luminance $40 \mathrm{~cd} \mathrm{~m}^{-2}$, refresh rate $60 \mathrm{~Hz}$ ) placed $\sim 10 \mathrm{~cm}$ away from the eye contralateral to the recording site or virus injection site. The Go and No-Go stimuli were vertically and horizontally oriented gratings, respectively. In each trial, the vertically (horizontally) oriented grating was static during the waiting period and then drifting rightward (upward) during the answer period. The Go and No-Go trials were randomly interleaved.

Behavioral task. Mice were water-deprived for 2 days before the behavioral training. During behavioral experiments, the mouse was head-fixed and sat in an acrylic tube within a training box. Tongue licks were detected by the interruption of an infrared beam or a capacitance touch sensor, and the delivery of water was controlled by a peristaltic valve (Kamoer). The mice went through a habituation phase and a conditioning phase before learning the Go/No-Go task. For habituation ( 2 days), the mouse learned to lick from a custom-made lickspout to get water reward every $4 \mathrm{~s}$. For conditioning (2-3 days), the mouse was trained to lick in response to a vertically oriented grating stimulus. The grating was static for $0.7 \mathrm{~s}$ or $0.5 \mathrm{~s}$ (waiting period) and then drifting for $2.2 \mathrm{~s}$ or $2.4 \mathrm{~s}$ (answer period). If a lick was detected during the answer period, the mouse was rewarded with $5.5 \mu \mathrm{l}$ of water. For Go/No-Go task, the grating stimulus in each trial was static during the waiting period and drifting during the answer period. For some groups of mice (Fig. 3, Fig. 4, Fig. 5, Supplementary Figs. 3-8 and Supplementary Fig. 11), the durations of waiting period and answer period were $0.7 \mathrm{~s}$ and $2.2 \mathrm{~s}$, respectively. For other groups of mice (Fig. 6, Supplementary Fig. 9 and Supplementary Fig. 10), the durations of waiting period and answer period were $0.5 \mathrm{~s}$ and $2.4 \mathrm{~s}$, respectively. Licking during the waiting period was neither rewarded nor punished. For a Go stimulus, if a lick was detected during the answer period, the mouse was rewarded with $5.5 \mu \mathrm{l}$ of water upon lick detection (hit). The mouse was neither rewarded nor punished for a miss (no lick during the answer period of Go stimulus), CR (no lick during the answer period of No-Go stimulus) or FA (lick during the answer period of No-Go stimulus). During the intertrial interval (ITI), the screen was blank and licking was punished by a timeout period of $4 \mathrm{~s}$. Licking during the 4-s timeout period triggered another 4-s timeout unless no lick was detected during the timeout period or the accumulated timeout exceeded $20 \mathrm{~s}$. Each mouse performed the task for $1 \mathrm{~h}$ in each session.

In a subset of behavioral sessions, we recorded images of the facial area ipsilateral to the $\mathrm{V} 1$ recording site with a point gray camera $(30 \mathrm{~Hz}$ frame rate) and a $780 \mathrm{~nm}$ longpass filter. Infrared LEDs $(840 \mathrm{~nm})$ were used to illuminate the face of the mouse. 
Optogenetic stimulation. Optical activation of ChR2 (ChrimsonR) was induced by blue (red) light. Optical silencing by Jaws activation was induced by red light. A blue laser $(473 \mathrm{~nm})$ or a red laser $(635 \mathrm{~nm})$ (Shanghai Laser \& Optics Century Co.) was connected to an output optical fiber and the laser was controlled by a stimulus generator (Master 9, A.M.P.I.).

For measuring synaptic inputs from $\mathrm{OFC}$ axons to $\mathrm{V} 1$ neurons during slice recording experiments, blue light ( $1 \mathrm{~ms}$ duration) was delivered through a $40 \times 0.8$ NA water immersion lens at a power of $50 \mathrm{~mW}$.

To manipulate the activity of OFC axons in V1 for in vivo recording or behavioral experiments, we used a zoom fiber collimator to focus the laser beam ( $\sim 600 \mu \mathrm{m}$ diameter) on $\mathrm{V} 1$ or on the center of the marked skull region above V1. A shield was mounted on the mouse's head to prevent leakage of laser light to the eyes or to the screen.

For in vivo extracellular recording of V1 neurons with optogenetic manipulation, trials with and without laser stimulation were interleaved. Laser stimulation covered the duration of stimulus presentation. For V1 recordings with laser stimulation during Go trials, the laser was turned on 100-ms before the onset of visual stimulus (Fig. $4 \mathrm{a}-\mathrm{d}$ ). For V1 recordings with laser stimulation during No-Go trials, the laser was turned on 100 -ms before stimulus onset for some mice (Supplementary Fig. 6a-d) and at stimulus onset for other mice (Supplementary Fig. $6 \mathrm{e}-\mathrm{j}$ ). Laser was at a power of $5 \mathrm{~mW}$ at collimator output for blue laser and $10 \mathrm{~mW}$ for red laser.

For behavioral experiments in which the $\mathrm{OFC}$ to $\mathrm{V} 1$ projection was optogenetically inactivated or SST interneurons in V1 were activated, laser-off and laser-on blocks ( 20 trials/block) were interleaved in each session. In laser-on blocks, laser stimulation was applied during No-Go trials and Go trials in two separate groups of mice, respectively. During trials with laser stimulation, the laser was turned on 100-ms before stimulus onset, and turned off at stimulus offset. Laser was at a power of 10 or $15 \mathrm{~mW}$ at collimator output.

For fiber photometry recording of OFC stimulation-induced V1 activity, $100-150$ pulses of blue laser light (500 ms duration, interval at $10 \mathrm{~s}$ ) were delivered to the optical fiber implanted in the OFC.

For phototagging of the V1-projecting OFC neurons, red laser light $(15 \mathrm{~mW})$ was applied on the surface of cortical area above the OFC ipsilateral to the virus injection site in V1. We delivered 400 light pulses (each 5-ms long) at $0.5 \mathrm{~Hz}$.

Histology. At least 2 weeks after the tracer/virus injection in V1 or virus injection in the OFC, the mouse was deeply anesthetized with sodium pentobarbital $\left(120 \mathrm{mg} \mathrm{kg}^{-1}\right)$ and was perfused with $60 \mathrm{ml}$ saline followed by $60 \mathrm{ml}$ paraformaldehyde (PFA, 4\%). Brains were collected, fixed in $4 \%$ PFA $\left(4^{\circ} \mathrm{C}\right)$ overnight, and then transferred to $30 \%$ sucrose in phosphate-buffered saline (PBS) until equilibration. Brain sections $(40 \mu \mathrm{m})$ were cut using a cryostat (Microm). The floating sections were incubated with Hoechst $(2 \mu \mathrm{M}$, Thermo Fisher Scientific) in PBS for $10 \mathrm{~min}$. The sections were rinsed in PBS for $10 \mathrm{~min}$, mounted onto glass slides and coverslipped with VECTASHIELD Antifade Mounting Medium (Vector Laboratories, H-1000). Fluorescence images were taken with a Nikon A1 (Nikon Co. Ltd.) confocal microscope or the VS120 (Olympus). Images were analyzed with ImageJ (NIH, US).

Data analysis. Analyses were performed in MATLAB. For the behavioral experiments, hit rate was computed as $N_{\text {hits }} /\left(N_{\text {hits }}+N_{\text {misses }}\right)$, where $N_{\text {hits }}$ and $N_{\text {misses }}$ are the numbers of hit and miss trials, respectively. FA rate $=N_{\mathrm{FAs}} /\left(N_{\mathrm{FAs}}+\right.$ $\left.N_{\mathrm{CRs}}\right)$, and CR rate $=N_{\mathrm{CRs}} /\left(N_{\mathrm{FAs}}+N_{\mathrm{CRs}}\right)$, in which $N_{\mathrm{FAs}}$ and $N_{\mathrm{CRs}}$ are the numbers of FA and CR trials, respectively. The behavioral discriminability was quantified as norminv(hit rate) - norminv(FA rate), in which norminv is the inverse of the cumulative normal function ${ }^{64}$. For behavioral experiments in which the OFC to V1 projection was optogenetically inactivated or SST interneurons in V1 were activated (Fig. 6), we computed hit rate, CR rate or discriminability using all trials (including laser-off and laser-on trials) in a session.

For each trial of Go/No-Go task, we computed lick latency as the time of first lick within $1 \mathrm{~s}$ after stimulus onset. The lick latency in each session was quantified as the median of first lick latency across trials. For behaving mice used for V1 or OFC recordings, the lick latency (V1 recording: $476.3 \pm 17.5 \mathrm{~ms}$, s.e.m.; OFC recording: $447.2 \pm 18.3 \mathrm{~ms}$, s.e.m.) was close to $0.5 \mathrm{~s}$ even if the waiting period was $0.7 \mathrm{~s}$. For the following analysis of neuronal responses during the waiting period, we thus used the responses within the first $0.5 \mathrm{~s}$ of the waiting period. To determine whether a V1 neuron was responsive to visual stimulus, we computed the baseline response during the $0.2 \mathrm{~s}$ before stimulus onset and the evoked firing rate during the waiting period of stimulus presentation for each trial. Those V1 neurons in which the evoked responses during the waiting period were larger than 0.5 spike/s and were significantly higher than the baseline response $(P<0.05$, Wilcoxon signed rank test) were used in the analysis. For the responses to No-Go stimulus during the waiting period, we divided the trials into $\mathrm{CR}$ and FA conditions, and computed a modulation index (MI) as $\left(R_{C R}-R_{F A}\right) /\left(R_{C R}+\mathrm{R}_{\mathrm{FA}}\right)$, in which $R_{C R}$ and $R_{F A}$ represented responses for CR and FA trials, respectively. For the analysis of MI, we only included neurons from those sessions in which the number of FA trials $>15$. Statistical significance of the MI of each cell was determined by comparing the waiting-period responses to No-Go stimulus between CR and FA trials with Wilcoxon rank sum test. The selectivity of V1 responses to the Go and No-Go stimuli was evaluated by a selectivity index (SI), defined as $\left(R_{G o}-R_{N o-G o}\right) /\left(R_{G o}+\right.$
$R_{N o-G o}$, in which $R_{G o}$ and $R_{N o-G o}$ are firing rates to the Go and No-Go stimuli during the waiting period, respectively.

For the experiments with optogenetic inactivation of OFC projection to $\mathrm{V} 1$ in behaving mice, we estimated the rate change of V1 neurons induced by laser stimulation in Go trials or No-Go trials. For Go trials, the rate change was computed as $\left(R_{\text {laser_on }}-R_{\text {laser_off }}\right) / R_{\text {laser_offs }}$ where $R_{\text {laser_on }}$ and $R_{\text {laser_off }}$ represented waiting-period firing rates to Go stimulus with and without laser stimulation, respectively. For CR condition in No-Go trials, the rate change was computed as $\left(R_{C R \_l a s e r \_o n}-R_{C R \_l a s e r \_o f f}\right) / R_{C R \_l a s e r \_o f f}$ where $R_{C R \_l a s e r \_o n}$ and $R_{C R \_l a s e r \_o f f}$ represented waiting-period firing rates to No-Go stimulus in CR trials with and without laser stimulation, respectively. For FA condition in No-Go trials, the rate change was computed as $\left(R_{F A \_l a s e r \_o n}-R_{F A_{-} \text {laser_off }}\right) / R_{F A \_l a s e r \_o f f}$ where $R_{F A \_l a s e r \_o n}$ and $R_{F A \_l a s e r \_o f f}$ represented waiting-period firing rates to No-Go stimulus in FA trials with and without laser stimulation, respectively. Because some mice had few FA trials due to a high behavioral performance, we only included V1 neurons from those sessions in which the numbers of FA trials in laser-on and laser-off conditions were both $>15$.

For extracellular recordings of V1 neurons from anesthetized or awake mice not performing behavioral task, we first estimated the RFs of neurons by crosscorrelating the responses with the sparse noise stimuli ${ }^{65}$. For the responses to oriented drifting gratings, spike rate to each stimulus was calculated by averaging the responses during the drifting period overall trials. For the responses without laser stimulation, we calculated the $t$ statistic (mean evoked rate divided by s.e.m.) for the responses to the preferred orientation ${ }^{66}$. For the responses in laser-off and laser-on conditions, respectively, we computed a global measure of orientation selectivity index (OSI) as:

$$
\text { OSI }=\sqrt{\left(\sum_{i}\left(R\left(\theta_{\mathrm{i}}\right) \sin \left(2 \theta_{\mathrm{i}}\right)\right)\right)^{2}+\left(\sum_{i}\left(R\left(\theta_{\mathrm{i}}\right) \cos \left(2 \theta_{\mathrm{i}}\right)\right)\right)^{2}} / \sum_{i} R\left(\theta_{\mathrm{i}}\right),
$$

where $\theta_{\mathrm{i}}$ is the angle of the drifting direction of the grating and $R\left(\theta_{\mathrm{i}}\right)$ is the response at angle $\theta_{\mathrm{i}}$. Only those units with OSI $>0.08$ (sensitive to orientation ${ }^{67}$ ), $t>2$ (visually responsive ${ }^{66}$ ), and peak evoked firing rate $>2 \mathrm{~Hz}$ during laser-off condition were included in the subsequent analyses. To estimate the effect of activating OFC axons in V1 on the response amplitude of V1 neurons, we computed a rate change index as $\left(R_{\text {laser_on }}-R_{\text {laser_off }}\right) /\left(R_{\text {laser_on }}+R_{\text {laser_off }}\right)$, in which $R_{\text {laser_on }}$ and $R_{\text {laser_off }}$ represented responses averaged overall orientations for laser-on and laser-off trials, respectively.

To obtain the normalized peri-stimulus time histogram (PSTH) over the population of neurons, the firing rates of each neuron were normalized by the maximum of the peak values of PSTHs in the Go and No-Go trials (or in hit, FA and CR trials), and were averaged across neurons.

We divided the responses of $\mathrm{V} 1$ neurons during the waiting period into an early component $(<100 \mathrm{~ms})$ and a late component $(>100 \mathrm{~ms})$. The SI and MI of each V1 neuron were computed for the early and the late components, respectively.

Based on the spike waveforms, we classified V1 neurons as broad-spiking and narrow-spiking cells, which correspond to putative excitatory and putative inhibitory neurons, respectively ${ }^{45,46}$. For each cell, the spikes were aligned by the trough and averaged, and the average waveform was interpolated ${ }^{46}$. We computed peak width as the width of the peak at half-maximum of the peak amplitude. Based on the distribution of the peak width (Supplementary Fig. 4), we defined a threshold at $0.35 \mathrm{~ms}$ to classify broad-spiking and narrow-spiking cells. The SI and MI were computed for broad-spiking and narrow-spiking cells, respectively.

To identify V1-projecting OFC neurons, we used the stimulus-associated spike latency test (SALT) ${ }^{49}$ to determine whether laser stimulation significantly changed the spike timing of neurons after stimulation onset. We also calculated Pearson's correlation coefficient between waveforms of spontaneous spikes and spikes during the 10-ms period after laser onset. A unit was identified as ChrimsonR-expressing neuron if $P<0.01$ for SALT test and waveform correlation coefficient $>0.9$. To determine the response latency relative to laser onset, we binned the spikes at 0.1 -ms resolution. For the PSTH within the 10-ms period after laser onset, we identified the time of peak firing rate. For each bin of laser-evoked response within the time of peak response, we tested the difference between the firing rate in this bin and that averaged over 10 -ms duration before laser onset $(t \text {-test })^{68}$. The latency was identified as the first time point after laser onset with $P<0.01$.

For OFC neurons recorded from behaving mice, we computed the baseline firing rates during the $0.2 \mathrm{~s}$ before stimulus onset and the responses to visual stimuli during the waiting period. Those neurons in which the waiting-period responses were significantly different from the baseline responses $(P<0.05$, Wilcoxon signed rank test) were used in the analysis. We computed MI for V1projecting OFC neurons using the same equation for V1 neurons. For OFC neurons, we also computed a response index (RI) for the responses to Go stimulus in hit trials and the responses to No-Go stimulus in CR (or FA) trials, respectively. $\mathrm{RI}$ was defined as $\left(\mathrm{R}_{\text {evoked }}-\mathrm{R}_{\text {baseline }}\right) /\left(\mathrm{R}_{\text {evoked }}+\mathrm{R}_{\text {baseline }}\right)$, where $\mathrm{R}_{\text {evoked }}$ and $\mathrm{R}_{\text {baseline }}$ represented the waiting-period firing rate and the baseline rate, respectively.

We performed several analyses to examine whether spike rate of V1 neurons or V1-projecting OFC neurons during the waiting period is affected by licking. First, we calculated the Pearson's correlation coefficient between the spike rate and lick rate in FA trials. For V1 neurons (V1-projecting OFC neurons), those sessions with $>20(>5)$ FA trials in which licks occurred in the waiting period were used for this 
analysis. Correlation with $P<0.05$ was considered as significant. Second, we used licks in the waiting period to compute histogram of lick-triggered spikes. For V1projecting OFC neurons, we further divided the neurons into two groups, in which the firing rates during the waiting period in hit trials were lower $(\mathrm{RI}<0)$ and higher $(\mathrm{RI}>0)$ than the baseline rate, respectively. Histogram of lick-triggered spikes were computed for both groups of V1-projecting OFC neurons. Third, we analyzed the selectivity index and modulation index of V1 neurons (V1-projecting OFC neurons) using those trials in which no lick occurred within the first $0.5 \mathrm{~s}$ following stimulus onset.

For fiber photometry experiments to measure V1 activity induced by OFC stimulation, the value of fluorescence change $(\Delta F / F)$ was derived by calculating $\left(F-F_{0}\right) / F_{0}$, where $F_{0}$ is baseline fluorescence signal averaged over $2 \mathrm{~s}$ before laser stimulation. For each mouse, we computed the peak of $\Delta F / F$ values after laser onset and the latency of peak $\triangle F / F$. For fiber photometry experiments to measure the activity of SST interneurons from behaving mice, $F_{0}$ was computed using the signal averaged over $1 \mathrm{~s}$ before stimulus onset. To compute the responses to the No-Go stimulus in $\mathrm{CR}$ (or $\mathrm{FA}$ ) trials, we averaged the values of $\triangle F / F$ during the waiting period.

The orofacial movements of the mice were analyzed using the FaceMap software (www.github.com/MouseLand/FaceMap) ${ }^{47}$. For each mouse, the motion energy PCs during the waiting period between laser-off and laser-on conditions were compared.

Statistical analysis. Sample sizes were similar to others used in the field. No statistical method was used to predetermine sample size. The statistical analysis was performed using MATLAB or GraphPad Prism (GraphPad Software). Wilcoxon signed rank test (one-sided or two-sided), Wilcoxon two-sided rank sum test, one-way repeated measures ANOVA, two-way repeated measures ANOVA, or two-way ANOVA with mixed designed was used to determine the significance of the effect. Correlation values were computed using Pearson's correlation. Data were not collected in a blinded fashion. Unless otherwise stated, data were reported as mean \pm s.e.m.

Reporting summary. Further information on research design is available in the Nature Research Reporting Summary linked to this article.

\section{Data availability}

The data supporting the findings of this study are available within the Article and Supplementary Information files or available from the corresponding author upon reasonable request. The source data underlying Figs. ld, e, h, i, 2b-d, f, i-k, 3c-n, $4 b-d, f-p$, $5 \mathrm{c}-\mathrm{g}, 6 \mathrm{~b}, \mathrm{c}, \mathrm{e}$, Supplementary Figs. 1, 3, 4b-d, 5, 6, 7, 8f- $\mathrm{o}, 9 \mathrm{e}-\mathrm{h}, \mathrm{j}, \mathrm{k}, 10$ and 11 are provided as a Source Data file.

\section{Code availability}

The data acquisition and analysis code are available from the corresponding author upon reasonable request.

Received: 16 October 2019; Accepted: 14 May 2020;

Published online: 03 June 2020

\section{References}

1. Murray, E. A., O’Doherty, J. P. \& Schoenbaum, G. What we know and do not know about the functions of the orbitofrontal cortex after 20 years of crossspecies studies. J. Neurosci. 27, 8166-8169 (2007).

2. Schoenbaum, G., Roesch, M. R., Stalnaker, T. A. \& Takahashi, Y. K. A new perspective on the role of the orbitofrontal cortex in adaptive behaviour. Nat. Rev. Neurosci. 10, 885-892 (2009).

3. Rudebeck, P. H. \& Murray, E. A. The orbitofrontal oracle: cortical mechanisms for the prediction and evaluation of specific behavioral outcomes. Neuron 84, 1143-1156 (2014).

4. Thorpe, S. J., Rolls, E. T. \& Maddison, S. The orbitofrontal cortex: neuronal activity in the behaving monkey. Exp. Brain Res. 49, 93-115 (1983).

5. Schoenbaum, G. \& Eichenbaum, H. Information coding in the rodent prefrontal cortex. I. Single-neuron activity in orbitofrontal cortex compared with that in pyriform cortex. J. Neurophysiol. 74, 733-750 (1995).

6. Schoenbaum, G., Chiba, A. A. \& Gallagher, M. Orbitofrontal cortex and basolateral amygdala encode expected outcomes during learning. Nat Neurosci. 1, 155-159 (1998).

7. Tremblay, L. \& Schultz, W. Relative reward preference in primate orbitofrontal cortex. Nature 398, 704-708 (1999).

8. Wallis, J. D. \& Miller, E. K. Neuronal activity in primate dorsolateral and orbital prefrontal cortex during performance of a reward preference task. Eur. J. Neurosci. 18, 2069-2081 (2003).

9. Roesch, M. R. \& Olson, C. R. Neuronal activity related to reward value and motivation in primate frontal cortex. Science 304, 307-310 (2004).
10. Kennerley, S. W. \& Wallis, J. D. Evaluating choices by single neurons in the frontal lobe: outcome value encoded across multiple decision variables. Eur. J. Neurosci. 29, 2061-2073 (2009).

11. Kepecs, A., Uchida, N., Zariwala, H. A. \& Mainen, Z. F. Neural correlates, computation and behavioural impact of decision confidence. Nature 455, 227-231 (2008).

12. Padoa-Schioppa, C. \& Assad, J. A. Neurons in the orbitofrontal cortex encode economic value. Nature 441, 223-226 (2006).

13. Gallagher, M., McMahan, R. W. \& Schoenbaum, G. Orbitofrontal cortex and representation of incentive value in associative learning. J. Neurosci. 19, 6610-6614 (1999).

14. Pickens, C. L. et al. Different roles for orbitofrontal cortex and basolateral amygdala in a reinforcer devaluation task. J. Neurosci. 23, 11078-11084 (2003).

15. Pickens, C. L., Saddoris, M. P., Gallagher, M. \& Holland, P. C. Orbitofrontal lesions impair use of cue-outcome associations in a devaluation task. Behav. Neurosci. 119, 317-322 (2005).

16. Schoenbaum, G., Setlow, B., Nugent, S. L., Saddoris, M. P. \& Gallagher, M. Lesions of orbitofrontal cortex and basolateral amygdala complex disrupt acquisition of odor-guided discriminations and reversals. Learn Mem. 10, 129-140 (2003).

17. Izquierdo, A., Suda, R. K. \& Murray, E. A. Bilateral orbital prefrontal cortex lesions in rhesus monkeys disrupt choices guided by both reward value and reward contingency. J. Neurosci. 24, 7540-7548 (2004).

18. McDannald, M. A., Saddoris, M. P., Gallagher, M. \& Holland, P. C. Lesions of orbitofrontal cortex impair rats' differential outcome expectancy learning but not conditioned stimulus-potentiated feeding. J. Neurosci. 25, 4626-4632 (2005).

19. Machado, C. J. \& Bachevalier, J. The effects of selective amygdala, orbital frontal cortex or hippocampal formation lesions on reward assessment in nonhuman primates. Eur. J. Neurosci. 25, 2885-2904 (2007).

20. West, E. A., DesJardin, J. T., Gale, K. \& Malkova, L. Transient inactivation of orbitofrontal cortex blocks reinforcer devaluation in macaques. J. Neurosci. 31, 15128-15135 (2011)

21. Rudebeck, P. H. \& Murray, E. A. Dissociable effects of subtotal lesions within the macaque orbital prefrontal cortex on reward-guided behavior. J. Neurosci. 31, 10569-10578 (2011).

22. Burke, K. A., Franz, T. M., Miller, D. N. \& Schoenbaum, G. The role of the orbitofrontal cortex in the pursuit of happiness and more specific rewards. Nature 454, 340-344 (2008).

23. Takahashi, Y. K. et al. The orbitofrontal cortex and ventral tegmental area are necessary for learning from unexpected outcomes. Neuron 62, 269-280 (2009).

24. McDannald, M. A., Lucantonio, F., Burke, K. A., Niv, Y. \& Schoenbaum, G. Ventral striatum and orbitofrontal cortex are both required for model-based, but not model-free, reinforcement learning. J. Neurosci. 31, 2700-2705 (2011).

25. Ostlund, S. B. \& Balleine, B. W. Orbitofrontal cortex mediates outcome encoding in Pavlovian but not instrumental conditioning. J. Neurosci. 27, 4819-4825 (2007)

26. Rudebeck, P. H., Saunders, R. C., Prescott, A. T., Chau, L. S. \& Murray, E. A. Prefrontal mechanisms of behavioral flexibility, emotion regulation and value updating. Nat. Neurosci. 16, 1140-1145 (2013).

27. Namboodiri, V. M. K. et al. Single-cell activity tracking reveals that orbitofrontal neurons acquire and maintain a long-term memory to guide behavioral adaptation. Nat. Neurosci. 22, 1110-1121 (2019).

28. Saddoris, M. P., Gallagher, M. \& Schoenbaum, G. Rapid associative encoding in basolateral amygdala depends on connections with orbitofrontal cortex. Neuron 46, 321-331 (2005)

29. Takahashi, Y. K. et al. Expectancy-related changes in firing of dopamine neurons depend on orbitofrontal cortex. Nat. Neurosci. 14, 1590-1597 (2011)

30. Schultz, W. \& Dickinson, A. Neuronal coding of prediction errors. Annu. Rev. Neurosci. 23, 473-500 (2000).

31. Takahashi, Y. K. et al. Neural estimates of imagined outcomes in the orbitofrontal cortex drive behavior and learning. Neuron 80, 507-518 (2013).

32. Zingg, B. et al. Neural networks of the mouse neocortex. Cell 156, 1096-1111 (2014).

33. Cavada, C., Company, T., Tejedor, J., Cruz-Rizzolo, R. J. \& Reinoso-Suarez, F The anatomical connections of the macaque monkey orbitofrontal cortex. A review. Cereb. Cortex 10, 220-242 (2000).

34. Carmichael, S. T. \& Price, J. L. Sensory and premotor connections of the orbital and medial prefrontal cortex of macaque monkeys. J. Comp. Neurol. 363, 642-664 (1995).

35. Gilbert, C. D. \& Li, W. Top-down influences on visual processing. Nat. Rev. Neurosci. 14, 350-363 (2013).

36. Zhang, S. et al. Selective attention. Long-range and local circuits for top-down modulation of visual cortex processing. Science 345, 660-665 (2014).

37. Winkowski, D, E et al. Orbitofrontal cortex neurons respond to sound and activate primary auditory cortex neurons. Cereb. Cortex 28, 868-879 (2017). 
38. Manita, S. et al. A top-down cortical circuit for accurate sensory perception. Neuron 86, 1304-1316 (2015).

39. Fiser, A. et al. Experience-dependent spatial expectations in mouse visual cortex. Nat. Neurosci. 19, 1658-1664 (2016).

40. Leinweber, M., Ward, D. R., Sobczak, J. M., Attinger, A. \& Keller, G. B. A sensorimotor circuit in mouse cortex for visual flow predictions. Neuron 95, 1420-1432 e1425 (2017).

41. Poort, J. et al. Learning enhances sensory and multiple non-sensory representations in primary visual cortex. Neuron 86, 1478-1490 (2015).

42. Makino, H. \& Komiyama, T. Learning enhances the relative impact of topdown processing in the visual cortex. Nat. Neurosci. 18, 1116-1122 (2015).

43. Wall, N. R., Wickersham, I. R., Cetin, A., De La Parra, M. \& Callaway, E. M. Monosynaptic circuit tracing in vivo through Cre-dependent targeting and complementation of modified rabies virus. Proc. Natl Acad. Sci. USA 107, 21848-21853 (2010).

44. Pfeffer, C. K., Xue, M., He, M., Huang, Z. J. \& Scanziani, M. Inhibition of inhibition in visual cortex: the logic of connections between molecularly distinct interneurons. Nat. Neurosci. 16, 1068-1076 (2013).

45. Bakhurin, K. I. et al. Differential encoding of time by prefrontal and striatal network dynamics. J. Neurosci. 37, 854-870 (2017).

46. Zhu, Y., Qiao, W., Liu, K., Zhong, H. \& Yao, H. Control of response reliability by parvalbumin-expressing interneurons in visual cortex. Nat. Commun. $\mathbf{6}$, 6802 (2015).

47. Stringer, C. et al. Spontaneous behaviors drive multidimensional, brainwide activity. Science 364, 255 (2019).

48. Tervo, D. G. et al. A designer AAV variant permits efficient retrograde access to projection neurons. Neuron 92, 372-382 (2016).

49. Kvitsiani, D. et al. Distinct behavioural and network correlates of two interneuron types in prefrontal cortex. Nature 498, 363-366 (2013).

50. Makino, H., Hwang, E. J., Hedrick, N. G. \& Komiyama, T. Circuit mechanisms of sensorimotor learning. Neuron 92, 705-721 (2016).

51. Sachidhanandam, S., Sreenivasan, V., Kyriakatos, A., Kremer, Y. \& Petersen, C. C. Membrane potential correlates of sensory perception in mouse barrel cortex. Nat. Neurosci. 16, 1671-1677 (2013).

52. Kwon, S. E., Yang, H., Minamisawa, G. \& O'Connor, D. H. Sensory and decision-related activity propagate in a cortical feedback loop during touch perception. Nat. Neurosci. 19, 1243-1249 (2016).

53. Vélez-Fort, M. et al. A circuit for integration of head- and visual-motion signals in layer 6 of mouse primary visual cortex. Neuron 98, 179-191 (2018).

54. Chubykin, A. A., Roach, E. B., Bear, M. F. \& Shuler, M. G. A cholinergic mechanism for reward timing within primary visual cortex. Neuron 77 , 723-735 (2013).

55. Zhou, J., Jia, C., Feng, Q., Bao, J. \& Luo, M. Prospective coding of dorsal raphe reward signals by the orbitofrontal cortex. J. Neurosci. 35, 2717-2730 (2015).

56. Khan, A. G. et al. Distinct learning-induced changes in stimulus selectivity and interactions of GABAergic interneuron classes in visual cortex. Nat. Neurosci. 21, 851-859 (2018).

57. McDannald, M. A., Jones, J. L., Takahashi, Y. K. \& Schoenbaum, G. Learning theory: a driving force in understanding orbitofrontal function. Neurobiol. Learn. Mem. 108, 22-27 (2014).

58. Berendse, H. W., Galis-de Graaf, Y. \& Groenewegen, H. J. Topographical organization and relationship with ventral striatal compartments of prefrontal corticostriatal projections in the rat. J. Comp. Neurol. 316, 314-347 (1992).

59. Cooch, N. K. et al. Orbitofrontal lesions eliminate signalling of biological significance in cue-responsive ventral striatal neurons. Nat. Commun. 6, 7195 (2015).

60. Pascoli, V. et al. Stochastic synaptic plasticity underlying compulsion in a model of addiction. Nature 564, 366-371 (2018).

61. Sharpe, M. J. \& Schoenbaum, G. Back to basics: making predictions in the orbitofrontal-amygdala circuit. Neurobiol. Learn. Mem. 131, 201-206 (2016).

62. Jo, Y. S. \& Mizumori, S. J. Y. Prefrontal regulation of neuronal activity in the ventral tegmental area. Cereb. Cortex 26, 4057-4068 (2016).

63. Groman, S. M. et al. Orbitofrontal circuits control multiple reinforcementlearning processes. Neuron 103, 734-746 (2019).
64. Andermann, M. L., Kerlin, A. M. \& Reid, R. C. Chronic cellular imaging of mouse visual cortex during operant behavior and passive viewing. Front. Cell. Neurosci. 4, 3 (2010).

65. Jones, J. P. \& Palmer, L. A. The two-dimensional spatial structure of simple receptive fields in cat striate cortex. J. Neurophysiol. 58, 1187-1211 (1987).

66. Hoy, J. L. \& Niell, C. M. Layer-specific refinement of visual cortex function after eye opening in the awake mouse. J. Neurosci. 35, 3370-3383 (2015).

67. Xu, X., Ichida, J., Shostak, Y., Bonds, A. B. \& Casagrande, V. A. Are primate lateral geniculate nucleus (LGN) cells really sensitive to orientation or direction? Vis. Neurosci. 19, 97-108 (2002).

68. Xu, M. et al. Basal forebrain circuit for sleep-wake control. Nat. Neurosci. 18, $1641-1647$ (2015)

\section{Acknowledgements}

We thank M. M. Poo for comments on the manuscript. We thank Y. Li and W. Xu for technical assistance and Y.-N. Dou for help with slice recordings. This work was supported by the Strategic Priority Research Program of Chinese Academy of Sciences (grant No. XDB32010200), Shanghai Municipal Science and Technology Major Project (grant No. 2018SHZDZX05), and the National Natural Science Foundation of China (31571079, 31771151).

\section{Author contributions}

D.L. and H.Y. conceived and designed the experiments. D.L. performed and organized all the experiments. J.D. and Y.-G.S. performed whole-cell recording experiments. D.L. Z.-Y.Z., Z.Z., T.Y., and H.Y. analyzed the data. H.Y. wrote the manuscript with inputs from all authors. All authors read and revised the manuscript.

\section{Competing interests}

The authors declare no competing interests.

\section{Additional information}

Supplementary information is available for this paper at https://doi.org/10.1038/s41467020-16609-7.

Correspondence and requests for materials should be addressed to H.Y.

Peer review information Nature Communications thanks Sylvain Crochet and other anonymous reviewers for their contributions to the peer review of this work.

Reprints and permission information is available at http://www.nature.com/reprints

Publisher's note Springer Nature remains neutral with regard to jurisdictional claims in published maps and institutional affiliations.

(c) (i) Open Access This article is licensed under a Creative Commons Attribution 4.0 International License, which permits use, sharing, adaptation, distribution and reproduction in any medium or format, as long as you give appropriate credit to the original author(s) and the source, provide a link to the Creative Commons license, and indicate if changes were made. The images or other third party material in this article are included in the article's Creative Commons license, unless indicated otherwise in a credit line to the material. If material is not included in the article's Creative Commons license and your intended use is not permitted by statutory regulation or exceeds the permitted use, you will need to obtain permission directly from the copyright holder. To view a copy of this license, visit http://creativecommons.org/ licenses/by/4.0/.

(C) The Author(s) 2020 